\title{
SOX9-induced Generation of Functional Astrocytes Supporting Neuronal Maturation in an All-human System
}

\author{
Katrien Neyrinck ${ }^{1}$ (D) Johanna Van Den Daele ${ }^{1} \cdot$ Tim Vervliet $^{2} \cdot$ Jonathan De Smedt $^{1} \cdot$ Keimpe Wierda $^{3} \cdot$ Melissa Nijs $^{1} \cdot$ \\ Tom Vanbokhoven ${ }^{1}$. Astrid D'hondt ${ }^{1}$ • Mélanie Planque ${ }^{4,5}$. Sarah-Maria Fendt ${ }^{4,5}$ • Pei-Yu Shih ${ }^{6}$. Frederik Seibt ${ }^{6}$. \\ Juan Pita Almenar ${ }^{6}$. Mohamed Kreir ${ }^{6}$ • Devesh Kumar ${ }^{6}$ • Vania Broccoli ${ }^{7,8}$ • Geert Bultynck ${ }^{2}$. Andreas Ebneth ${ }^{6}$. \\ Alfredo Cabrera-Socorro $^{6} \cdot$ Catherine Verfaillie $^{1}$
}

Accepted: 29 April 2021 / Published online: 12 May 2021

(C) The Author(s) 2021

\begin{abstract}
Astrocytes, the main supportive cell type of the brain, show functional impairments upon ageing and in a broad spectrum of neurological disorders. Limited access to human astroglia for pre-clinical studies has been a major bottleneck delaying our understanding of their role in brain health and disease. We demonstrate here that functionally mature human astrocytes can be generated by $S O X 9$ overexpression for 6 days in pluripotent stem cell (PSC)-derived neural progenitor cells. Inducible (i)SOX9astrocytes display functional properties comparable to primary human astrocytes comprising glutamate uptake, induced calcium responses and cytokine/growth factor secretion. Importantly, electrophysiological properties of iNGN2-neurons co-cultured with iSOX9-astrocytes are indistinguishable from gold-standard murine primary cultures. The high yield, fast timing and the possibility to cryopreserve iSOX9-astrocytes without losing functional properties makes them suitable for scaled-up production for high-throughput analyses. Our findings represent a step forward to an all-human iPSC-derived neural model for drug development in neuroscience and towards the reduction of animal use in biomedical research.
\end{abstract}

Keywords Astrocytes $\cdot$ Pluripotent stem cells $\cdot$ Differentiation protocol $\cdot$ Genome engineering $\cdot$ All-human co-culture system

\section{Introduction}

Astrocytes are the most abundant cell type in the brain and are crucial for healthy central nervous system functioning [1]. Dysfunction of astrocytes has been identified to play a key role in brain-ageing and neurodegenerative diseases,

Katrien Neyrinck and Johanna Van Den Daele contributed equally to this work.

Katrien Neyrinck

katrien.neyrinck@kuleuven.be

Johanna Van Den Daele

johanna.vandendaele@kuleuven.be

Catherine Verfaillie

catherine.verfaillie@kuleuven.be

1 Stem Cell Institute, Department of Development and Regeneration, KU Leuven, Leuven 3000, Belgium

2 Laboratory of Molecular and Cellular Signalling, Department of Cellular and Molecular Medicine, KU Leuven, Leuven, Belgium

3 Electrophysiology Expert Unit, VIB-KU Leuven Center for Brain \& Disease Research, Leuven 3000, Belgium including Alzheimer's disease (AD) and amyotrophic lateral sclerosis (ALS) $[2,3]$. In vivo, astrocytes provide trophic and metabolic neuronal support $[4,5]$ as well as maintaining proper synapse and blood-brain-barrier (BBB) functioning [6, 7]. In addition, astrocytes take up excessive extracellular ions and neurotransmitters preventing neuronal toxicity $[8,9]$. These
4 Laboratory of Cellular Metabolism and Metabolic Regulation, Department of Oncoloy, KU Leuven and Leuven Cancer Institute (LKI), Leuven 3000, Belgium

5 Laboratory of Cellular Metabolism and Metabolic Regulation, VIB-KU Leuven Center for Cancer Biology, VIB, Leuven 3000, Belgium

6 Division of Janssen Pharmaceutica, Janssen Research \& Development, Beerse 2340, Belgium

7 Division of Neuroscience, IRCCS, San Raffaele Scientific Hospital, 20132 Milan, Italy

8 Institute of Neuroscience, National Research Council (CNR), 20129 Milan, Italy 
functions of astrocytes are also key for in vitro maturation of neurons. Especially in vitro cultured neurons derived from pluripotent stem cells (PSCs) need astrocytic support to acquire typical neuronal electrophysiological properties so they become amenable for functional studies [10-13]. To achieve PSC-derived neuronal maturation, most studies have used primary rodent-derived astrocytes. However, rodent astrocyte functions are not fully conserved in higher order mammals. In human or primate brains for instance, more elaborate neuronal networks require greater numbers and more complex astrocytes [7, 14]. Therefore, human PSC-derived astrocytes would preferably be used. Initially, PSC-derived astrocytes were generated by mimicking developmental astroglial differentiation using only growth factors and/or small molecules [15-18]. However, such protocols are very labor intensive and lengthy, ranging from 75 to 600 days. To bypass this time-consuming process, transcription factor $(\mathrm{TF})$ overexpression can be used to speed up the differentiation process, as has been done for neurons ( $N G N 2$ overexpression), for example [10]. In 2015, Caiazzo et al. found that overexpression of the TFs NFI-A, NFI-B and SOX9 was sufficient to convert mouse fibroblasts into astrocyte-like cells [19]. Three recent studies have used this approach to create human astrocytes from PSCs, by inducing expression of either NFI-A and SOX9 or NFI-B and SOX9 in PSCs, or NFI-A as the sole TF in PSCderived neural progenitor cells (NPCs) [20-22]. During mouse development, $\mathrm{SOX} 9$ is already expressed at E10.5 and binds to astrocyte (and oligodendrocyte) specific genes, such as NFI-A that becomes expressed at E11.5 [23, 24]. Many SOX9 targeted regions also contain NFI-motifs, to which NFI-A binds. SOX9 and NFI-A synergistically activate gene expression during the gliogenic switch [24]. During further astrocyte (but not oligodendrocyte) maturation steps, SOX9 and NFI-A remain expressed activating mature astrocytic genes, with a crucial role of SOX9 as its loss prevents astrogenesis $[25,26]$. Because of the central role of SOX9 in these developmental steps and based on our previous findings that nearly $100 \%$ pure oligodendrocytes can be generated from PSCs when $S O X 10$ (without co-factors) is overexpressed in NPCs [27, 28], we here hypothesized that induced expression of SOX9 without the need for the co-factors, NFI-A or $N F I-B$, might suffice to create astrocytes.

We demonstrated that $S O X 9$ overexpression by doxycycline in iPSC-derived NPCs is indeed sufficient to generate a stable astrocyte phenotype already from 6 days after doxycycline treatment and remains stable for at least 4 weeks following removal of doxycyline. Transcriptome analysis indicated that the induced (i)SOX9-astrocytes attain a maturation state intermediate between freshly isolated pre- and post-natal human astrocytes. iSOX9-astrocytes displayed robust glutamate uptake, calcium responses towards ATP and acetylcholine (Ach) and secreted cytokines/growth factors after stimulation with TNF- $\alpha$ and/or IL-1 $\beta$. Most importantly, we demonstrate that iSOX9-astrocytes support synchronous activity of iNGN2-PSC neuronal progeny to levels higher than seen in gold-standard rat neuronal primary cultures. The high yield (> 100 astrocytes per PSC), the possibility of cryopreservation and fast timing ( \pm 40 days) of the protocol makes iSOX9astrocytes a useful cell source for scaled up-production for high-throughput analyses of human iPSC-derived neural models and drug development, while sustaining the $3 \mathrm{R}$ principle related to animal use in biomedical research.

\section{Materials and Methods}

\section{Cell Culture and Genome-engineering}

Normal donor iPSCs (SIGi001-A, purchased from Sigma) were cultured at $37^{\circ} \mathrm{C}$ and $5 \% \mathrm{CO}_{2}$ on matrigel-coated 6-well plates (Cornig) with E8 flex medium (Gibco) and passaged twice a week using $0.5 \mathrm{mM}$ EDTA (Gibco). First, the AAVS1 recombination master cell line was created as decribed before [29]. Briefly, iPSCs were detached using accutase (Sigma) and single cell suspensions $\left(10^{6}\right.$ cells) were resuspended in $100 \mu 1$ Nucleofector Solution ${ }^{\mathrm{TM}}$ (Lonza) with $5 \mu \mathrm{g}$ of a donor plasmid and $2 \mu \mathrm{g}$ mRNA coding for the left and right ZFNs. The donor plasmid contains homology arms flanking the ZFN-mediated double strand break with in between the homology arms a combination of GFP and hygromycin/ thymidine kinase (TK) located between FRT and FRT3 sites. Following nucleofection with the Amaxa nucleofector device (program F16), single cells were plated on matrigel-coated plates in $\mathrm{mTeSR}^{\mathrm{TM}} 1$ medium (Stemcell Technologies) supplemented with rock inhibitor (Y-27632, VWR). When small colonies appeared, hygromycin (Hygromycin B, Thermoscientific) selection was performed by gradually increasing hygromycin concentrations from 50 to $300 \mu \mathrm{g} / \mathrm{ml}$. Single colonies were manually picked and cultured separately. Quality control consisted of 5' junction PCR to assure insertion inside the AAVS1 locus and southern blot to detect possible random integration events.

iPSC clones that passed all QC, were subsequently subjected to recombinase-mediated cassette exchange (RMCE) to replace the GFP-Hygro-TK cassette with a cassette containing FRT and FRT3 sites in identical orientation, a promotorless puromycin cassette for gene trapping and an inducible TET-ON system for overexpression of SOX9 (SIGi001-A-20 cells). Nucleoporation and subsequent selection was again performed as described by Ordovas et al. [29]. Correctly recombined cells were selected by initial positive selection using puromycin (Sigma; 120 to $300 \mathrm{ng} / \mu \mathrm{l}$ ) followed by negative selection with $0.5 \mu \mathrm{M}$ Fialuridine (FIAU, Sigma).

iCell astrocytes were commercially bought from Cellular Dynamics and are human iPSC-derived astrocytes. fHA were 
commercially bought from ScienCell and were derived from fetal cortex. Both commercially bought astocytes were cultured in DMEMF12 Glutamax ${ }^{\mathrm{TM}}$ (Gibco) supplemented with G5 (Gibco) and HB-EGF (Peprotech; $10 \mathrm{ng} / \mathrm{mL}$ ).

\section{Generation of Lentiviral Particles}

The plasmids needed for the generation of lentiviral vector particles are psPAX2 (Addgene \#12260), pMD2.G (Addgene \#12259), FUW-M2rtTA (Addgene \#20342) and the plasmids encoding SOX9, NFI-A and NFI-B under a TET-ON promotor were kindly provided by prof. Broccoli from the San Raffaele Scientific Institute in Milan. Lenti-X HEK293T cells (ClonTech) were plated at a density of $125000 \mathrm{cells} / \mathrm{cm}^{2}$ in HEK medium consisting of DMEM high glucose Glutamax ${ }^{\mathrm{TM}}$ (Gibco) supplemented with $10 \%$ FBS (ThermoFisher), $100 \mathrm{U} / \mathrm{ml}$ Penicillin-Streptomycin (Gibco) and 1X sodium pyruvate (Gibco). The next day when cells reached $90 \%$ confluency, they were transduced with $4 \mu \mathrm{g}$ pMD2.G, $12 \mu \mathrm{g}$ psPAX2 and $12 \mu \mathrm{g}$ plasmid encoding for either M2rtTA, SOX9, NFI-A or NFI-B with the use of FuGENE® HD Transfection Reagent (Promega). After six hours, medium was changed to regular HEK medium and lentiviral vector particles were collected, filtered using a $0.45 \mu \mathrm{m}$ Steriflip (Millipore) and stored at $-80{ }^{\circ} \mathrm{C} 36 \mathrm{~h}$ later.

\section{Lentiviral Screen for Astrocyte Generation}

Single cell suspensions of iPSCs were plated at 150000 cells $/ \mathrm{cm}^{2}$ in matrigel-coated plates in $\mathrm{mTeSR}^{\mathrm{TM}} 1$ medium supplemented with 1X Revitacell (Gibco). Medium was changed the next day to neural induction (NI) medium consisting of NMM supplemented with $1 \mu \mathrm{M}$ LDN193,189 (Miltenyi) and $10 \mu \mathrm{M} \mathrm{SB} 431542$ (Tocris). NMM consists of a 1:1 mixture of Neurobasal medium (Gibco) and DMEMF12 Glutamax ${ }^{\mathrm{TM}}$ supplemented with $0.5 \mathrm{X}$ Glutamax ${ }^{\mathrm{TM}}$ (Gibco), $50 \mathrm{U} / \mathrm{ml}$ PenicillinStreptomycin, 0.5X B27 (Gibco), 0.5X N2 (Gibco), $0.5 \mathrm{X}$ MEM-NEAA (Gibco), 0.5X sodium pyruvate, $0.025 \%$ human insulin (Sigma) and $50 \mu \mathrm{M} 2$ mercaptoethonal (Gibco). NI medium was changed daily for 12 consecutive days. Afterwards, cells were detached using accutase and plated single cell at a density of 65 000 cells $/ \mathrm{cm}^{2}$ in NMM with $20 \mathrm{ng} / \mathrm{ml} \mathrm{bFGF}$ (Peprotech) and $1 \mathrm{X}$ Revitacell. The next day, different combinations of the TF-containing lentiviral vectors (NFI-A, NFI-B, SOX9, NFI-A + SOX9, NFI-B + SOX9, NFI-A + NFI-B + $S O X 9$ ) combined with an m2rtTA lentiviral vector were added to the wells. After 1 day, medium was changed to DMEMF12 Glutamax ${ }^{\mathrm{TM}}$ supplemented with $1 \mathrm{X}$ N2 and
$3 \mu \mathrm{g} / \mathrm{ml}$ doxycycline (Doxycycline hyclate, Sigma). Medium was changed every other day for 7 days.

\section{iSOX9-astrocyte Differentiation}

Single cell suspensions of SIGi001-A-20 iPSCs were plated at density of 150000 cells $/ \mathrm{cm}^{2}$ on matrigel-coated plates in $\mathrm{mTeSR}^{\mathrm{TM}} 1$ medium supplemented with $1 \mathrm{X}$ Revitacell. The next day, medium was changed to NI medium with daily medium changes for 12 consecutive days. On day 12 , cells were detached using accutase and single cell suspension was plated on matrigel coated plates at 65 000 cells $/ \mathrm{cm}^{2}$ in NMM supplemented with $20 \mathrm{ng} / \mathrm{ml} \mathrm{bFGF}$ and $1 \mathrm{X}$ Revitacell. The following day, medium was replaced to maturation medium. Maturation medium consists of a 1:1 mixture of Neurobasal medium and DMEMF12 Glutamax $^{\mathrm{TM}}$ supplemented with $1 \mathrm{X}$ N2, 1X sodium pyruvate, $1 \mathrm{X}$ Glutamax ${ }^{\mathrm{TM}}, 0.5 \mathrm{mM} \mathrm{N}$-acetyl-cysteine (Sigma), $0.1 \mathrm{mM}$ dbcAMP (Sigma), $10 \mathrm{ng} / \mathrm{ml}$ CNTF (Peprotech), 10 $\mathrm{ng} / \mathrm{ml} \mathrm{BMP} 4$ (Peprotech) and $5 \mathrm{ng} / \mathrm{ml} \mathrm{HB-EGF}$ (Peprotech). For the initial 6 days, maturation medium was supplemented with $3 \mu \mathrm{g} / \mathrm{ml}$ doxycycline and replaced every other day. From then onwards, cells were maintained in maturation medium without doxycycline and medium was changed twice a week. When the cells were $>90 \%$ confluent, they were harvested with accutase and replated at $20000 \mathrm{cells} / \mathrm{cm}^{2}$ in maturation medium.

\section{RT-qPCR}

RNA was isolated with the Quick-RNA Microprep Kit (Zymo research) according to the manufacturer's instructions. cDNA was made starting from $500 \mathrm{ng}$ RNA using the SuperScript ${ }^{\mathrm{TM}}$ III First-Strand Synthesis System (Thermofisher) kit, followed by RT-qPCR using Platinum SYBR Green qPCR SupermixUDG (Thermofisher). Primer sequences can be found in Table 1.

\section{Flow Cytometry}

Cells were detached using accutase and fixed for $15^{\prime}$ at RT in $4 \%$ PFA (Sigma). Cells were washed with PBS and permeabilized with $100 \%$ methanol (VWR) for $30^{\prime}$ at $4^{\circ} \mathrm{C}$. After washing with PBS, cells were incubated with primary antibodies for $45^{\prime}$ at $4^{\circ} \mathrm{C}$, washed and incubated with secondary antibodies for $30^{\prime}$ at $4^{\circ} \mathrm{C}$. After washing with PBS, flow cytometry was performed using the FACSCanto HTS device (BD Biosciences) acquiring 10,000 cells and data analyzed using FACSDiva software (version 6.1.2). Note that all centrifugation steps were done for $5^{\prime}$ at 600 rcf. List of antibodies can be found in Table 2. As control, samples only stained with secondary antibodies were used. 
Table 1 RT-qPCR primer sequences

\begin{tabular}{lll}
\hline Gene & Forward primer & Reverse primer \\
\hline OCT4 & GATGGCGTACTGTGGGCCC & TGGGACTCCTCCGGGTTTTG \\
$P A X 6$ & AGGCCCTGGAGAAAGAGTTT & TTTGGCTGCTAGTCTTTCTCG \\
NESTIN & TCAGCTTTCAGGACCCCAAG & TGGGAGCAAAGATCCAAGACG \\
TUBB3 & CCTCCGTGTAGTGACCCTT & GGCCTTTGGACATCTCTTCAG \\
SOX9 & CGTTCTTCACCGACTTCCTC & CTGGGCAAGCTCTGGAG \\
$N F I-A$ & CTTCATGCCATCCACTTGACT & CTTTACCCAGCACATCCTCTAC \\
$N F I-B$ & GATCATTGTGGCTTGGACTTC & GCCACATCATATCACAGTAT \\
CD 44 & CAGT \\
S $100 B$ & GAGATGCTGTAGCGACCATT & GACACCATGGACAAGTTTTGG \\
GFAP & TGGCCCTCATCGACGTTTC & ATGTTCAAAGAACTCGTGGCA \\
ALDHIL1 & GTCCCCCACCTAGTTTGCAG & TAGTCGTTGGCTTCGTGCTT \\
\hline
\end{tabular}

\section{Immunostaining and Fluorescence Microscopy}

Cells were fixed with $4 \%$ PFA for $15^{\prime}$ at RT. After washing with PBS, cells were blocked and permeabilized for 20' at RT with $5 \%$ goat serum (Dako) and $0.1 \%$ Triton X-100 (Sigma), all diluted in PBS. Next, primary antibodies were diluted in $5 \%$ goat serum and added to the cells at $4{ }^{\circ} \mathrm{C}$. The next day, cells were washed with PBS, and secondary antibodies diluted in Dako REAL Antibody Diluent (Dako) were added to the cells for 45' at RT. Afterwards, the cytoplasm was stained using Cellmask (Thermo Fisher Scientific, diluted 1/2500 in PBS) for 30' followed by a nuclear staining with Hoechst33342 (Sigma, diluted 1/1000 in Dako REAL Antibody diluent) for 10', both at RT. The list of antibodies can be found in Table 2. Images were acquired using the Operetta High Content Imaging System (PerkinElmer). Consecutive image analysis was performed using automatic and unbiased object segmentation and counting in each channel using the image analysis platform Columbus
(PerkinElmer). In short, nuclei (Hoechst staining) was the first sub-cellular structure to be segmented and used as a reference point for cytoplasm segmentation (Cellmask staining). Both segmented cell regions were selected for each separate immunofluorescence experiment and each time between 2500 and 14,000 cells were considered. Within the selected cell population, the mean fluorescence intensity was used to set a threshold for calculating the percentage of positive cells for each marker. In addition, the integrated density was calculated by dividing the mean fluorescence intensity by the area of the cytoplasm (in $\mu \mathrm{m}^{2}$ ).

\section{RNA Sequencing}

\section{RNA Quality Control}

RNA concentration and purity were determined spectrophotometrically using the Nanodrop ND-1000 (Nanodrop

Table 2 List of antibodies used

\begin{tabular}{llll}
\hline & Antibody & Dilution & Company \\
\hline Primary antibodies & Rabbit anti-S100B & $1 / 100$ (Flow cytometry) & Abcam \\
& & $1 / 200$ (IF) & \\
& Rabbit anti-EAAT1 & $1 / 1000$ (Flow cytometry) $1 / 200$ & Abcam \\
& Rat anti-GFAP & $1 / 1000$ (IF) & Thermo Fisher \\
& & & Scientific \\
& Mouse anti-ALDH1L1 & $1 / 500$ (IF) & Abcam \\
Gecondary & Antibodies & $1 / 500$ & Thermo Fisher \\
& Alexa488 & & Scientific \\
& Goat anti-rat Alexa555 & $1 / 500$ & Thermo Fisher \\
& Goat anti-mouse & $1 / 500$ & Scientific \\
& Alexa555 & & Thermo Fisher \\
& & & Scientific \\
\hline
\end{tabular}


Technologies) and RNA integrity was assessed using a Bioanalyser 2100 (Agilent) and a 5300 Fragment Analyzer (Agilent).

\section{Library Preparation}

Per sample, an amount of 100 ng of total RNA was used as input. For samples which contained less than 100ng, all RNA was used. Using the Illumina TruSeq ${ }^{\circledR}$ Stranded mRNA Sample Prep Kit (protocol version: Part \# 1000000040600 v00 October 2017), poly-A containing mRNA molecules were purified from the total RNA input using poly-T oligoattached magnetic beads. In a reverse transcription reaction using random primers, RNA was converted into first strand cDNA and subsequently converted into double-stranded cDNA in a second strand cDNA synthesis reaction using DNA Polymerase I and RNAse $H$. The cDNA fragments were extended with a single ' $A$ ' base to the 3 ' ends of the bluntended cDNA fragments after which multiple indexing adapters were ligated introducing different barcodes for each sample. Finally, enrichment PCR was carried out to enrich those DNA fragments that have adapter molecules on both ends and to amplify the amount of DNA in the library.

\section{Sequencing}

Sequence-libraries of each sample were equimolarly pooled and sequenced on Illumina HiSeq 4000 (2 lanes, 75 bp, Single Reads) at the VIB Nucleomics core (www.nucleomics.be).

\section{Preprocessing}

Low quality ends and adapter sequences were trimmed off from the Illumina reads with FastX 0.0.14 and Cutadapt 1.15 [30] (http://hannonlab.cshl.edu/fastx_toolkit/index. html). Subsequently, small reads (length $<35 \mathrm{bp}$ ), polyAreads (more than $90 \%$ of the bases equal A), ambiguous reads (containing N), low-quality reads (more than $50 \%$ of the bases $<$ Q25) and artifact reads (all but three bases in the read equal one base type) were filtered using FastX 0.0.14 and ShortRead 1.40.0 [31]. With Bowtie2 2.3.3.1 we identified and removed reads that align to phix illumina [32].

\section{Mapping}

The preprocessed reads were aligned with STAR aligner $\mathrm{v} 2.5 .2 \mathrm{~b}$ to the reference genome of Homo sapiens (GRCh38) [33]. Default STAR aligner parameter settings were used, except for '-outSAMprimaryFlag OneBestScore -twopassMode Basic -alignIntronMin 50 -alignIntronMax 500000 -outSAMtype BAM SortedByCoordinate'. Using Samtools 1.5 , reads with a mapping quality smaller than 20 were removed from the alignments [34].

\section{Counting}

The number of reads in the alignments that overlap with gene features were counted with featureCounts 1.5.3 [35]. Following parameters were chosen: -Q 0 -s 2 -t exon -g gene_id. We removed genes for which all samples had less than 1 count-per-million. Raw counts were further corrected within samples for GC-content and between samples using full quantile normalization, as implemented in the EDASeq package from Bioconductor [36]. All fastq and supplementary files were uploaded to NCBI-GEO under accession number GSE162892.

\section{Differential Gene Expression}

With the EdgeR 3.24.3 package of Bioconductor, a negative binomial generalized linear model (GLM) was fitted against the normalized counts [37]. We did not use the normalized counts directly, but worked with offsets. Differential expression was tested for with a GLM likelihood ratio test, also implemented in the EdgeR package. The resulting p-values were corrected for multiple testing with BenjaminiHochberg to control the false discovery rate [38].

\section{Gene Set Enrichment Analysis}

To detect enriched functional gene sets, we used the online tool Webgestalt (http://www.webgestalt.org/) with gene sets from the "biological process" gene ontology functional database. Genes were ranked by the signed log of the $\mathrm{P}$ values from the differential expression analysis. The sign comes from the differentially expressed log2-values. We used the HGNC symbols for gene identifiers. We limited our analysis to gene sets with less than or equal to 500 genes and more than or equal to 20 genes.

\section{Inclusion Data Zhang et al., Li et al., Tchieu et al.}

Fastq files from Zhang et al. [39], Li et al. [21] and Tchieu et al. [22] were downloaded from the European Nucleotide Archive. Quality control of raw reads was assessed with FastQC version 0.11.5. Cutadapt version 1.9.1 was used to trim residual adapter sequences, bases with Phred-scores lower than 20, poly A $\{10\}$ tail sequences, and reads shorter than 20 bases. STAR version 020201 was used to assemble a genome index, using the human genome fasta files (GRCh38.92) and GTF file from Ensembl. Subsequently, STAR version 020201 was used to align the trimmed reads to the human genome. Raw read count matrices were generated with FeatureCounts (from RsubRead). The raw count matrices from Zhang et al. and from this study were merged into one matrix. Merged exon lengths were calculated with GTFtools and subsequently used for TPM and log2 (TPM + 
1) calculation. The Combat algorithm from the sva $R$ package was used for batch effect correction. Principal Component Analysis was performed on the corrected $\log 2(\mathrm{TPM}+1)$ values using the $\mathrm{R}$ stats package.

\section{Glutamate Uptake}

DIV33-48 iSOX9-astrocytes and fHA were seeded in matrigel-coated plates at 20000 cells $/ \mathrm{cm}^{2}$. The next day, medium was replaced with DMEMF12 Glutamax ${ }^{\mathrm{TM}}$ medium which contains $50 \mu \mathrm{M}$ glutamate, supplemented with $1 \mathrm{X}$ $\mathrm{N} 2,1 \mathrm{X}$ sodium pyruvate, $0.5 \mathrm{mM} \mathrm{N}$-acetyl-cysteine, $0.1 \mathrm{mM}$ dbcAMP, $10 \mathrm{ng} / \mathrm{ml} \mathrm{CNTF,} 10 \mathrm{ng} / \mathrm{ml} \mathrm{BMP} 4$ and 5 $\mathrm{ng} / \mathrm{ml}$ HB-EGF. After 24, 48 and $72 \mathrm{~h}$, medium was collected for mass spectrometry.

Metabolite extraction was performed in a mixture ice-dry ice as described before $[40,41]$. A standard curve from 0 to $100 \mu \mathrm{mol} / \mathrm{L}$ of glutamic acid was prepared to determine the uptake/secretion of glutamic acid in media samples. A volume of $20 \mu \mathrm{L}$ of media samples and standards were resuspended in $800 \mu \mathrm{L}$ of methanol/water (5/3) (v/v) containing $0.6 \mu \mathrm{g} / \mathrm{ml}$ of glutaric acid as internal standard. Subsequently, a volume of $500 \mu \mathrm{L}$ of precooled chloroform was added to each sample. Samples were then vortexed for $10 \mathrm{~min}$ at $4{ }^{\circ} \mathrm{C}$ and centrifuged for other $10 \mathrm{~min}$ (max. speed, $4{ }^{\circ} \mathrm{C}$ ). After centrifugation, metabolites were separated in two phases: polar metabolites in the methanol/water (upper) phase and the lipid fraction in the chloroform (lower) phase. Following metabolites separation, polar metabolite phase containing glutamic acid was dried at $4{ }^{\circ} \mathrm{C}$ overnight using a vacuum concentrator. The samples were derivatized and measured as described before [42]. Briefly, polar metabolites were derivatized in $20 \mu \mathrm{L}$ of $20 \mathrm{mg} / \mathrm{ml}$ methoxyamine in pyridine per sample for $90 \mathrm{~min}$ at $37^{\circ} \mathrm{C}$. Subsequently, $15 \mu \mathrm{L}$ of N-(tert-butyldimethylsilyl)$\mathrm{N}$-methyl-trifluoroacetamide, with $1 \%$ tertbutyldimethylchlorosilane were added to $7.5 \mu \mathrm{L}$ of each derivative and incubated for $60 \mathrm{~min}$ at $60{ }^{\circ} \mathrm{C}$.

The metabolites were analyzed by gas chromatography (7890 A GC system) coupled to mass spectrometry (5975 C Inert MS system) from Agilent Technologies. Metabolites were separated with a DB35MS column $(30 \mathrm{~m}, 0.25 \mathrm{~mm}$, $0.25 \mu \mathrm{m}$ ) using a carrier gas flow of helium fixed at $1 \mathrm{ml} /$ min for the analysis of polar metabolites. A volume of $1 \mu \mathrm{L}$ of sample were injected in splitless mode with an inlet temperature set at $270{ }^{\circ} \mathrm{C}$. For the detection of polar metabolites, the gradient started at $100{ }^{\circ} \mathrm{C}$ for $1 \mathrm{~min}$ ramped to $105^{\circ} \mathrm{C}$ at $2.5^{\circ} \mathrm{C} / \mathrm{min}$, then to $240{ }^{\circ} \mathrm{C}$ at $3.5^{\circ} \mathrm{C} / \mathrm{min}$ and finally to $320^{\circ} \mathrm{C}$ at $22{ }^{\circ} \mathrm{C} / \mathrm{min}$. For the measurement of metabolites by mass spectrometry, the temperatures of the quadrupole and the source were set at 150 and $230{ }^{\circ} \mathrm{C}$, respectively. An electron impact ionization fixed at $70 \mathrm{eV}$ was applied and a selectedion monitoring (SIM) mode was used for the measurement of polar metabolites.
After the acquisition by GC-MS, a inhouse Matlab M-file was used to extract mass distribution vectors and integrated raw ion chromatograms. The natural isotopes distribution were also corrected using the method developed by Fernandez et al., 1996 [43]. The peak area was subsequently normalized to the internal standard: glutaric acid.

\section{ELISA}

DIV33-48 iSOX9-astrocytes from three independent differentiations and fHA were exposed for 5 days to either $10 \mathrm{ng} / \mathrm{ml}$ TNF- $\alpha$ (Peprotech), 100 ng/ml IL-6 (Peprotech), 10 ng/ml IL$1 \beta$ (Peprotech) or the combination of TNF- $\alpha$ and IL- $1 \beta$. In addition, an untreated condition was taken into account. After 5 days, medium was collected in duplicates for the iSOX9astrocytes and in triplicate for the fHA. The following analytes were measured using the LEGENDplex ${ }^{\mathrm{TM}}$ bead-based immunoassay according manufacturer's instructions: GM-CSF, HGF, IFN- $\alpha$, IFN- $\beta$, IL-10, IL-12, IL-1 $\alpha$, IL-1 $\beta$, IL-23, IL6 , LIF, PDGF-AA, TNF- $\alpha$.

\section{Calcium Imaging}

DIV33-48 iSOX9-astrocytes and fHA were plated in a round 4-chamber cover slip (100 000 cells/chamber) one week prior to the measurements. On the day of the recordings, cells were loaded with $1.25 \mu \mathrm{M}$ Fura-2 (Eurogentec) in modified Krebs solution $\left(150 \mathrm{mM} \mathrm{NaCl}, 5.9 \mathrm{mM} \mathrm{KCl}, 1.2 \mathrm{mM} \mathrm{MgCl}_{2}\right.$, 11.6 mM HEPES (pH 7.3), $11.5 \mathrm{mM}$ glucose and $1.5 \mathrm{mM}$ $\mathrm{CaCl}_{2}$; all purchased from Sigma) at RT in the dark. After 30 minutes, the cells were washed twice with the above modified Krebs solution followed by a $30^{\prime}$ ' incubation/de-esterification step at RT in the modified Krebs solution. During the recordings, an automated suction system was positioned to ensure complete medium change upon manual addition of the different stimuli. Each measurement was started by addition of 3 mM BAPTA (VWR) in the modified Krebs solution lacking $1.5 \mathrm{mM} \mathrm{CaCl}_{2}$, to chelate extracellular $\mathrm{Ca}^{2+}$. One minute later, the different stimuli, solubilized in modified Krebs solution without $\mathrm{CaCl}_{2}$ supplemented with $3 \mathrm{mM} \mathrm{BAPTA}$, were added and responses were measured. For quantifying ATP $(20 \mu \mathrm{M}$, Roche) and Ach ( $20 \mu \mathrm{M}$, Sigma) responses, the number of responding cells were counted as well as the AUC was determined. For GPN (200 $\mu \mathrm{M}$, Abcam), FCCP $(10 \mu \mathrm{M}$, Abcam), thapsigargin $(2 \mu \mathrm{M}$, Alomone labs) and ionomycin $(2 \mu \mathrm{M}$, Alomone labs), the area under the curve for each trace was calculated. Changes in Fura-2 fluorescence intensity were measured at $510 \mathrm{~nm}$ while alternating excitation at $340 \mathrm{~nm}$ and $380 \mathrm{~nm}$ utilizing a Zeiss Axio Observer Z1 Inverted Microscope equipped with a $20 \times$ air objective and a highspeed digital camera (Axiocam Hsm, Zeiss, Jena, Germany). Traces are shown as the ratio of emitted fluorescence of Fura2 (F340/F380). 


\section{Patch Clamping of Astrocytes}

DIV48 iSOX9-astrocytes and fHA were plated on a matrigel-coated $3 \mathrm{~cm}$ coverslip at a density of 8000 cells $/ \mathrm{cm}^{2}$ and whole-cell voltage clamp recordings were performed. The intracellular pipette solution contained $136 \mathrm{mM} \mathrm{KCl}, 18 \mathrm{mM}$ HEPES, $4 \mathrm{mM}$ Na-ATP, $4.6 \mathrm{mM}$ $\mathrm{MgCl}_{2}, 4 \mathrm{mM} \mathrm{K}_{2}$-ATP, $15 \mathrm{mM}$ creatine phosphate, $1 \mathrm{mM}$ EGTA and $50 \mathrm{U} / \mathrm{mL}$ phospocreatine kinase $(300 \mathrm{mOsm}$, $\mathrm{pH}$ 7.30). The extracellular solution used during recordings contained $140 \mathrm{mM} \mathrm{NaCl}, 2.4 \mathrm{mM} \mathrm{KCl}, 2 \mathrm{mM} \mathrm{CaCl}_{2}$, $2 \mathrm{mM} \mathrm{MgCl}$, $10 \mathrm{mM}$ HEPES, $10 \mathrm{mM}$ glucose $(300$ mOsm, pH 7.30). Cultured astrocytes were whole-cell voltage clamped at $-70 \mathrm{mV}$ using a double EPC-10 amplifier (HEKA Elektronik, Lambrecht/Pfalz, Germany) under control of Patchmaster v2 $\times 32$ software (HEKA Elektronik). Currents were recorded at $20 \mathrm{~Hz}$ and lowpass filtered at $3 \mathrm{kHz}$ when stored. Pipettes were pulled using a Sutter P-1000 and resistance ranged from 3 to $5 \mathrm{M}$ $\Omega$. The series resistance was compensated to $75-85 \%$. Cells with series resistances above $15 \mathrm{M} \Omega$ were excluded for analysis. I/V-traces were generated using a step protocol $(-120$ to $+120 \mathrm{mV}, 20 \mathrm{mV}$ steps $)$ from a holding potential of $-70 \mathrm{mV}$. $\mathrm{Na}^{+}$and $\mathrm{K}^{+}$currents were measured at the beginning and end of the voltage steps, respectively. Resting membrane potential $(\mathrm{Vm})$ was measured after the establishment of whole-cell configuration (in current clamp, Iholding $=0 \mathrm{pA}$ ). All recordings were performed at room temperature.

\section{Multi-electrode Array (MEA) Experiments}

Human iNGN2-neurons, generated by 4 days of doxycycline $(2 \mu \mathrm{g} / \mathrm{ml})$ addition to iNGN2-PSCs in NMM, were co-cultured with DIV40 iSOX9-astrocytes from 4 independent differentiations in 1:1 ratio and plated in NMM without doxycycline onto 48-well MEA plates (Axion BioSystems) pre-coated with $0.1 \%$ polyethyleneimine (Sigma). Spontaneous network activity was recorded for 5 minutes 37 days after seeding using the Axion Biosystems Maestro MEA at $37{ }^{\circ} \mathrm{C}$ and $5 \% \mathrm{CO}_{2}$. Data analysis was performed using AxIs software (Axion Biosystems Inc.). Active electrodes, (AEs; 16 electrodes per well) were defined as electrodes averaging more than 5 spikes per minute. Active wells were defined as those that have more than $30 \%$ of active electrodes. All wells that did not comply with these criteria were discarded. The threshold for spike detection was defined as $\geq 5.5$ fold the standard deviation of the RMS (root mean square) noise. To be able to calculate the synchronicity of the neuronal culture, at least $25 \%$ of the total electrode in a well should participate to the network bursts.

\section{Statistical Analysis}

All cell culture experiments were performed in triplicate (three independent differentiations; except for the lentiviral screen where only two independent differentiations were carried out) and values are represented as mean \pm SEM. For comparing means comprising one categorical variable, One-way ANOVA was performed with Bonferroni correction for multiple comparisons. Similarly, Two-way ANOVA was used if two categorical variables were taken into account and was performed with Bonferroni correction. Statistical significance was reached when $p \leq 0.05$. All data was processed via GraphPad Prism version 9.0.0 (GraphPad Software, San Diego, California, USA).

\section{Results}

\section{Six Days of SOX9 Overexpression in iPSC-derived NPCs Upregulates the Expression of Astrocyte-specific Proteins}

To test the hypothesis that overexpression of $S O X 9$ alone, without the co-factors NFI-A and NFI-B, at the NPC stage would be sufficient to induce an astrocyte fate, we performed a preliminary lentiviral vector-based screen. NPCs were generated from PSCs by dual SMAD inhibition. On DIV12, iPSC-derived NPCs were transduced with lentiviral vectors encoding for doxycycline-inducible SOX9, NFI-A or NFI-B, either alone or in combination. Inducible overexpression of the TFs was confirmed via RT-qPCR (Figure S1). Flow cytometry (Figure S2b) and RT-qPCR (Figure S2c) one week after transduction demonstrated that overexpression of $S O X 9$ alone was sufficient to induce an astrocyte phenotype at the transcript and protein level. Early neuronal transcripts (TUBB3, PAX6, NESTIN) were not induced and pluripotency transcript $O C T 4$ did not increase after lentiviral transduction of any of the combinations (Figure S2c).

To ensure that all NPCs contain a single copy of the transgene and to avoid the theoretical possibility of insertional mutagenesis caused by lentiviral transduction, we next recombined a cassette encoding for $S O X 9$ controlled by a TET-ON promotor via recombinase-mediated cassette exchange (RMCE) in the AAVS1 locus as described in Ordovas et al., [29]. First, we introduced by homologous recombination using zinc finger nucleases (ZFNs) a GFPexpressing cassette flanked by two FRT-sites into the AAVS1 locus of iPSCs. We confirmed specific integration via flow cytometry, PCR and Southern Blot (Figure S2d and Figures S3a-b). Afterwards, we recombined with Flippase (Flp) an FRT-flanked cassette containing SOX9 controlled by a TET-ON promoter. After RMCE, GFP expression disappeared (Figure S2d) and doxycycline addition to the cells 
induced SOX9 transcript levels (Figure S2e). Genome-edited stem cells were tested by array comparative genomic hybridization $(\mathrm{aCGH})$ and showed no genome wide mutations (Figure S3c). In addition, embryoid body (EB) formation and Scorecard ${ }^{\circledR}$ analysis demonstrated pluripotency of the genome-engineered iSOX9-iPSCs (SIGi001-A-20) (Figure S3d).

Using flow cytometric analysis for S100B and EAAT1 and transcript analysis of astrocyte-related genes, we tested how many days of doxycycline-mediated $S O X 9$ overexpression was necessary and sufficient to create iSOX9-astrocytes (Fig. 1a-c). These studies demonstrated that a stable astrocyte fate was already obtained after 6 days of forced SOX9 overexpression. Specifically, when doxycycline was removed on day 6 and NPC-progeny reassessed by RT-qPCR 5 and 30 days later, $S 100 B$ transcript levels remained steady and day 30 levels were similar to those found in cultured fetal human astrocytes (fHA) (Fig. 1d). As expected, removal of doxycycline reduced transcript levels of $S O X 9$ (Fig. 1d). Transcript levels for GFAP were lower in iSOX9-astrocytes compared to fHA on day 6 of doxycyline treatment as well as 5 and 30 days after removal of doxycycline ( $p<0.0001)$ (Fig. 1d). Flow cytometry and immunostaining demonstrated that the fraction of cells positive for S100B and EAAT1 was similar to fHA in iSOX9-astrocytes 5 and 30 days after stopping doxycycline (Fig. 1e, f). Immunostaining also demonstrated that as for fHA, the majority of iSOX9-astrocytes stained positive for ALDH1L1 (Fig. 1f). Consistent with the RT-qPCR results, significantly fewer day 5 and day 30 iSOX9-astrocytes stained positive for GFAP and GFAP intensity was lower compared to fHA ( $\mathrm{p}<0.05)$ (Fig. 1f). Morphological analysis demonstrated that the roundness of iSOX9-cells decreased between 5 and 30 days of doxycycline removal, but fHA roundness was even smaller compared with day 30 iSOX9-astrocytes $(\mathrm{p}<$ 0.001) (Fig. 1g). A progressive decrease in proliferation of iSOX9-astrocyte progeny was observed from \pm day 20 after stopping doxycycline onwards (Fig. 1h). Per iPSC, 105.05 \pm 11.43 iSOX9-astrocytes could be generated. Moreover, iSOX9-astrocytes could be cryopreserved and did not lose their astrocytic phenotype upon thawing (Figure S4). In conclusion, a nearly $100 \%$ homogenous population of S100B and EAAT1 positive astrocyte-like cells can be generated by forced overexpression of $S O X 9$, as the sole TF, for only 6 days in iPSC-derived NPCs.

\section{iSOX9-astrocytes Display Functional Glutamate Handling, Electrophysiological Properties, Cytokine/growth Factor Secretion as well as Intracellular Calcium Responses}

The function of iSOX9-astrocytes was assessed between 15 and 30 days after doxycycline removal (DIV33-48) in comparison with fHA as positive control. As astrocytes are responsible for glutamate removal from the synapse to prevent glutamate-induced excitotoxicity, glutamate handling was assessed [44]. We incubated iSOX9-astrocytes and fHA with medium containing $50 \mu \mathrm{M}$ glutamate and measured glutamate concentration in the medium after 24,48 and $72 \mathrm{~h}$ by mass spectrometry. No significant differences in glutamate uptake were observed between iSOX9-astrocytes and fHA (Fig. 2a).

Patch clamp experiments were performed to characterize the electrophysiological properties of iSOX9-astrocytes in comparison with fHA. No differences were observed in resting membrane potential between iSOX9-astrocytes and fHA (Fig. 2b). However, cell capacitance measurements revealed a significant bigger cell size of iSOX9-astrocytes compared to fHA ( $<0.01)$ (Fig. 2b), in line with the morphometric analysis shown in Fig. 1g. As functional astrocytes express voltage-gated channels, we also measured $\mathrm{Na}^{+}$and $\mathrm{K}^{+}$currents at voltages ranging from $-120 \mathrm{mV}$ until $+160 \mathrm{mV}$. Results were corrected for cell size. Although we observed $\mathrm{Na}^{+}$currents in iSOX9-astroyctes, these were less pronounced compared to fHA (Fig. 2c). Outward $\mathrm{K}^{+}$currents were comparable between the iSOX9-astrocytes and fHA (Fig. 3c). Both $\mathrm{Na}^{+}$and $\mathrm{K}^{+}$currents were significantly lower in both iSOX9-astrocytes and fHA compared to what is commonly measured in neurons [45], further demonstrating that iSOX9astrocytes do not contain neural contaminants.

We also assessed the response of iSOX9-astroyctes to inflammatory stimuli (IL- 6 , TNF- $\alpha$, IL- $1 \beta$ or the combination of TNF- $\alpha$ and IL-1 $\beta$ ). After 5 days of stimulation, secretion of different cytokines and growth factors was assessed by a beadbased multiplex immunoassay for pro-inflammatory (GMCSF, TNF- $\alpha$, IL- $1 \alpha$, IL-1 $\beta$, IL-6, IL-12 and IL-23) and anti-inflammatory (IFN- $\alpha$, IFN- $\beta$, IL-10, HGF, LIF and PDGFaa) cytokines/growth factors. Data is presented as the fold change compared to untreated iSOX9-astrocytes or fHA. In general the cytokine/growth factor levels secreted by iSOX9-astrocytes and fHA were similar. Specifically, no IL$1 \alpha$, IFN- $\alpha$, IFN- $\beta$, HGF, IL- 12 and IL-10 response was observed in either iSOX9-astrocytes or fHA in response to any of the inflammatory stimuli. Exposure to IL- $1 \beta$, with or without TNF- $\alpha$, induced secretion of LIF, IL- 6 and GM-CSF by both iSOX9-astrocytes and fHA (Fig. 2d). Increased secretion of IL-23 was observed to all stimuli in iSOX9-astrocytes only $(\mathrm{p}<0.0001)$ and TNF- $\alpha$ treatment induced secretion of the protective growth factors LIF and PDGFaa by iSOX9-astrocytes, but not fHA $(p<0.05)$ (Fig. 2d). Finally, GM-CSF and IL-6 secretion induced by IL-1 $\beta+$ TNF- $\alpha$ was higher in fHA compared to iSOX9-astrocytes ( $\mathrm{p}<0.0001)$ (Fig. $2 \mathrm{~d}$ ).

As astrocyte communication is governed by $\mathrm{Ca}^{2+}$ signaling, we also compared single cell $\mathrm{Ca}^{2+}$ responses of iSOX9-astrocytes and fHA. First, two physiological 
a

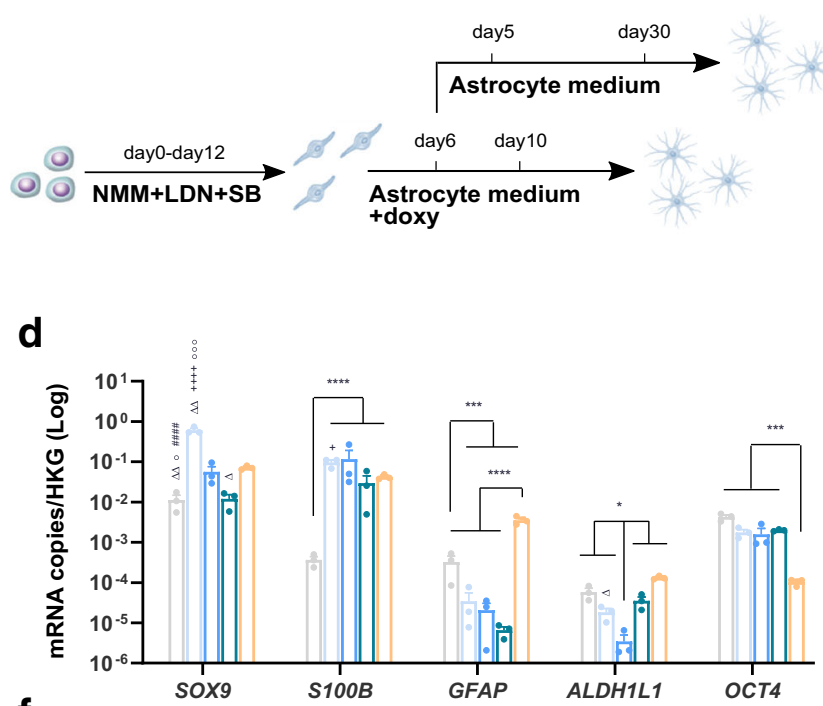

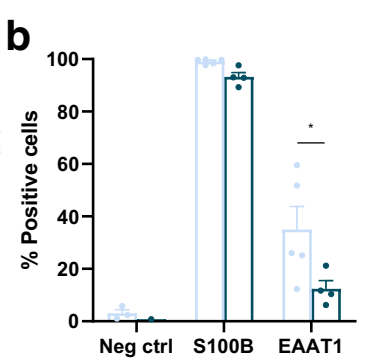

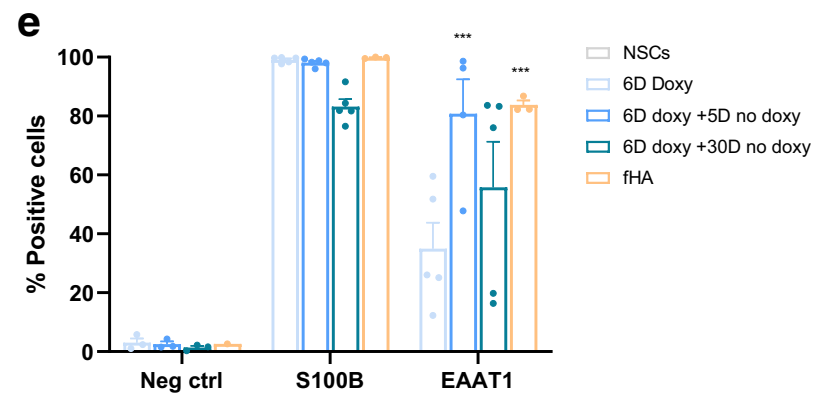

\section{C}

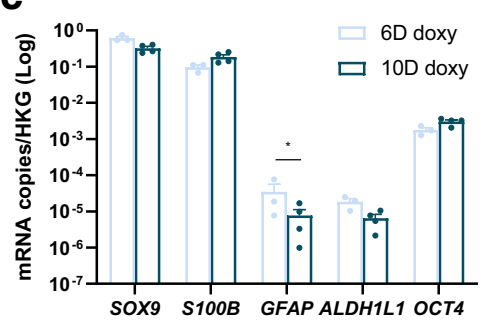

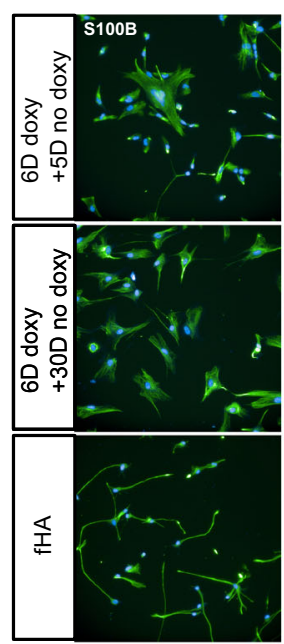

S100B

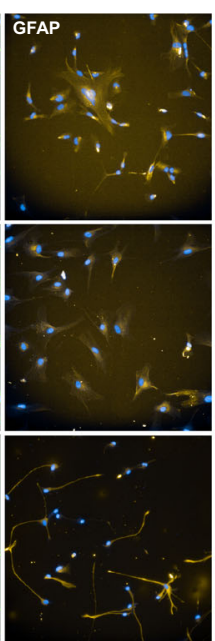

GFAP

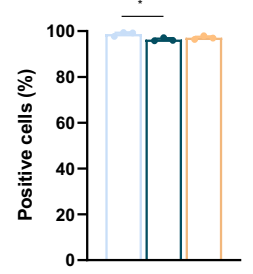

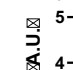

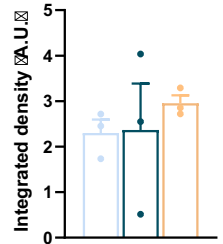

g
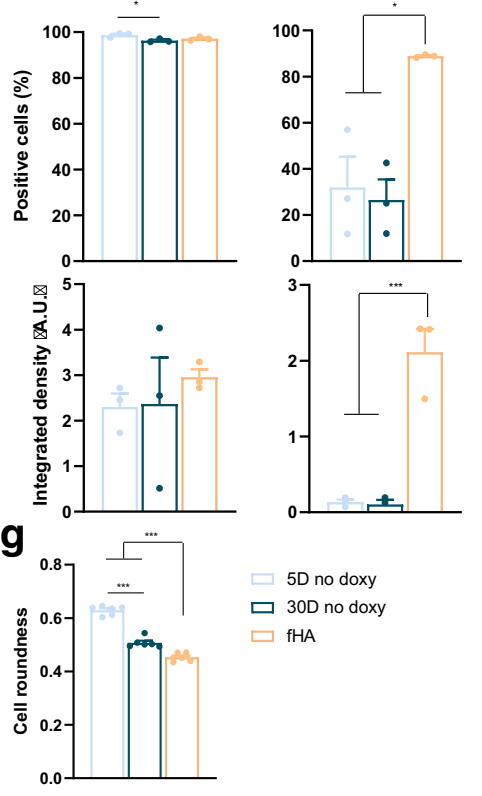

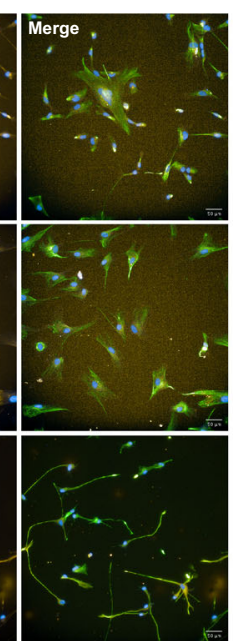

$\square$ D no doxy

$\square$ 30D no doxy

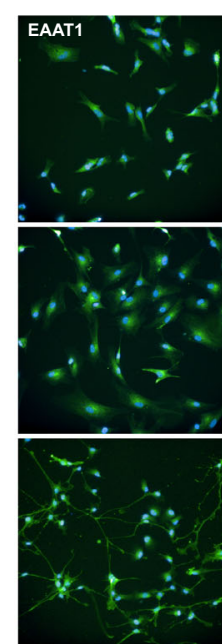

EAAT1
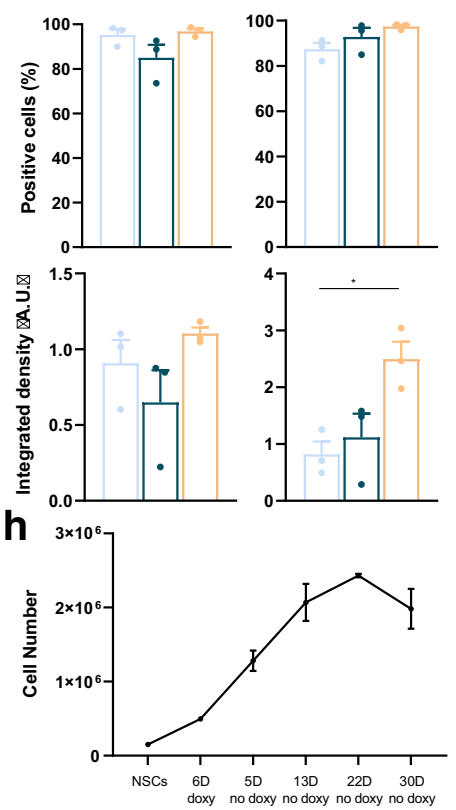

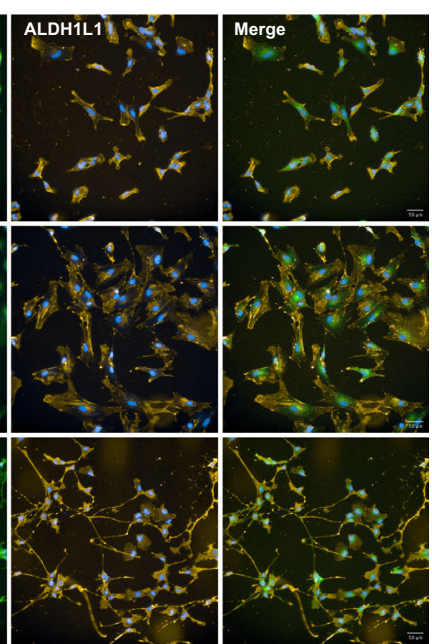

ALDH1L1

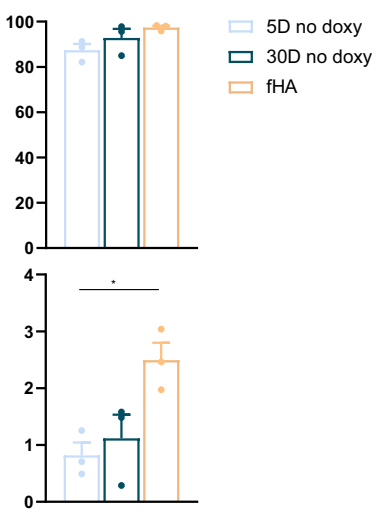

$$
\text { . }
$$


Fig. 1 Six days of SOX9 overexpression in iSOX9-derived NPCs upregulates the expression of astrocyte-specific proteins. a Overview optimization of iSOX9-astrocyte differentiation. First, the number of days to overexpress $S O X 9$ were tested by comparing 6 and 10 days of doxycycline exposure. Next, the effect of removing doxycycline was assessed. b Flow cytometry to assess the percentage of S100B and EAAT1 expressing cells after exposing iSOX9-derived NPCs for 6 and 10 days to doxycycline $(\mathrm{N}=4-5$ independent differentiations; $* \mathrm{p}<0.05)$. c RT-qPCR of $S O X 9$, S1OOB, GFAP, ALDH1L1 and OCT4 transcripts after exposing iSOX9-derived NPCs for 6 and 10 days to doxycycline $(\mathrm{N}=3-4$ independent differentiations; * $\mathrm{p}<0.05)$. d RT-qPCR of SOX9, S100B, GFAP, ALDH1L1 and OCT4 transcripts at different time points throughout the optimized differentiation protocol compared to fHA. $(\mathrm{N}=$ 3 independent differentiations; ${ }^{\Delta} \mathrm{p}<0.05$ versus fHA, ${ }^{\# \# \#} \mathrm{p}<0.0001$ versus $6 \mathrm{D}$ doxy, ${ }^{\circ} \mathrm{p}<0.05$ versus $5 \mathrm{D}$ no doxy, ${ }^{+} \mathrm{p}<0.05$ versus $30 \mathrm{D}$ no doxy). e Flow cytometry to assess the percentage of S100B and EAAT1 expressing cells at different time points throughout the optimized differentiation protocol compared with fHA $(\mathrm{N}=3-5$ independent differentiations; $* * * p<0.001$ versus $6 \mathrm{D}$ doxy). f Representative immunofluorescence images of fHA and iSOX9-astrocytes at 5 and 30 days after stopping doxycycline treatment for S100B (green), GFAP (red), EAAT1 (green) and ALDH1L1 (red) (scale bar: $50 \mu \mathrm{m}$ ). Quantification of the percentage of positive cells and integrated density was performed using Columbus Image analysis software (PerkinElmer) $(\mathrm{N}=3$ independent differentiations; $* \mathrm{p}<0.05, * * * \mathrm{p}<0.001)$. g Cell roundness quantification of fHA and iSOX9-astrocytes after stopping doxycycline treatment for 5 and 30 days $(\mathrm{N}=3$ independent differentiations; $* * * p<0.001)$. $\mathbf{h}$ Quantification of cell numbers throughout iSOX9-astrocyte differentiation ( $\mathrm{N}=3$ independent differentiations). All data represented as mean \pm SEM

inducers ATP and acetylcholine (Ach) were used to trigger intracellular $\mathrm{Ca}^{2+}$ release. The fraction of iSOX9astrocytes responding to ATP and Ach and the corresponding area under the curve (AUC) were similar to that of fHA (Fig. 3a, b). Also histamine and caffeine were used, but as they did not induce $\mathrm{Ca}^{2+}$ responses in either iSOX9astrocytes or fHA, data is not shown. Next, thapsigargin and ionomycin were used as triggers in order to measure the endoplasmic reticulum (ER) $\mathrm{Ca}^{2+}$ store content and total cellular $\mathrm{Ca}^{2+}$ content respectively. Thapsigargin induces the depletion of the ER $\mathrm{Ca}^{2+}$ store by blocking $\mathrm{Ca}^{2+}$ reuptake via the sarco/endoplasmic reticulum $\mathrm{Ca}^{2+}$ ATPase. Thapsigargin treatment induced a significantly lower $\mathrm{Ca}^{2+}$ response in iSOX9-astrocytes compared to fHA $(\mathrm{p}<0.01)$. However, the total cellular $\mathrm{Ca}^{2+}$ release triggered by ionomycin was similar in both astrocyte types (Fig. 3c). To assess $\mathrm{Ca}^{+}{ }^{+}$release related to other organelles besides the ER, cells were also exposed to glycyl-Lphenylalanine 2-naphthylamide (GPN) and carbonyl cyanide 4-(trifluoromethoxy)phenylhydrazone (FCCP). GPN triggers intracellular $\mathrm{Ca}^{2+}$ release by inducing the rupture of lysosomal membranes or by increasing both lysosomal and cytosolic $\mathrm{pH}$ resulting in $\mathrm{Ca}^{2+}$ release in an ERdependent manner [46]. FCCP exposure leads to collapse of the mitochondrial membrane potential and subsequent mitochondrial $\mathrm{Ca}^{2+}$ release which can then be amplified by ER $\mathrm{Ca}^{2+}$ release or by $\mathrm{Ca}^{2+}$ influx over the plasma membrane [47]. Exposure of iSOX9-astrocytes to both stimuli induced similar $\mathrm{Ca}^{2+}$ responses as seen for fHA (Fig. 3c).

Thus, iSOX9-astroyctes have the ability to handle glutamate, have electrophysiological properties, respond to inflammatory stimuli and show intracellular $\mathrm{Ca}^{2+}$ responses that are in general similar to fHA.

\section{Transcriptional Profiling of iSOX9-astrocytes Reveals Maturation After Stopping Doxycycline Treatment}

We performed RNA sequencing on iSOX9-astrocytes 5 (early; DIV23) and 30 (late; DIV48) days after stopping doxycycline treatment. Aside from the healthy donor derived SIGi001-A-20 iSOX9-astrocytes, we also included early and late iSOX9-astrocytes from an ALS patient-derived iPSC line (as described in Guo et al., [48]), genome-engineered in the same way to allow forced expression of $S O X 9$. We also included commercially available iPSC-derived astrocytes ("iCell" astrocytes) and fHA. Hierarchical clustering demonstrated that all iSOX9-astrocytes (independent of harvest day and donor origin) clustered separately from iCell astrocytes and cultured fHA. Within the group of iSOX9-astrocytes, early and late astrocytes clustered separately, again independent of the donor origin of the iPSC (Fig. 4a). Differential gene expression analysis was used to identify genes that were signficanly less or more expressed in early versus late iSOX9-astrocytes. Gene set enrichment analysis (GSEA) identified proliferation-related processes to be enriched in early astrocytes independent of the iPSC origin (Fig. 4b). Late iSOX9-astrocytes generated from both iPSC lines displayed an enrichment of astrocyte-related biological functions such as synapse organization and regulation of membrane potential and neurotransmitter levels. The GSEA analysis is therefore indicative of maturation of iSOX9-astrocytes after doxycycline removal.

To assess how well iSOX9-astrocytes approximate primary uncultured human astrocytes, we used transcriptome data of human fetal and postnatal brains published by Zhang et al. [39]. We also compared iSOX9 transcriptome data with data from $\mathrm{Li}$ et al. who created astrocytes by overexpression of SOX9 and NFIA directly in PSCs [21], and Tchieu et al. who overexpressed only NFIA directly in PSC-derived longterm neural stem cells [22]. Principle component analysis (PCA) of all genes is shown in Figure S5. As this PCA only captured $24.55 \%$ of genes in principle components 1 and 2 , we next identified genes that were at least 16 -fold differentially expressed between human primary brain-derived fetal or postnatal astrocytes from Zhang et al. This selection should identify astrocyte-specific genes that are linked to age and/or the development state of astrocytes, which is the factor we are interested in for comparing the different astrocyte 
a

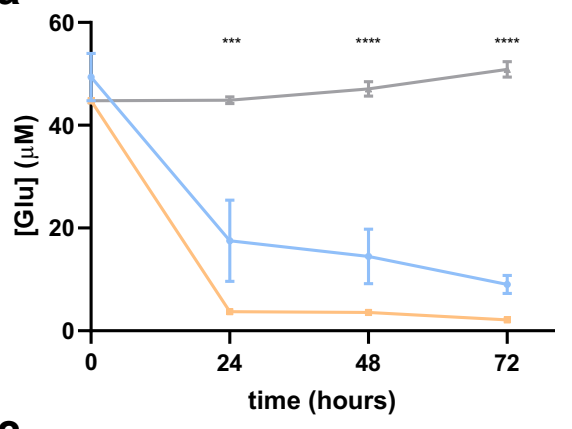

C

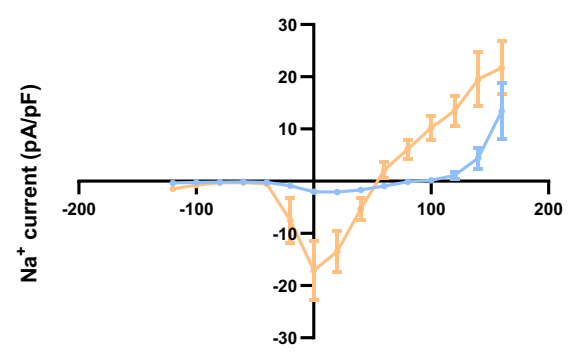

Voltage (mV)
- isox9

$=$ fHA

- No cells b
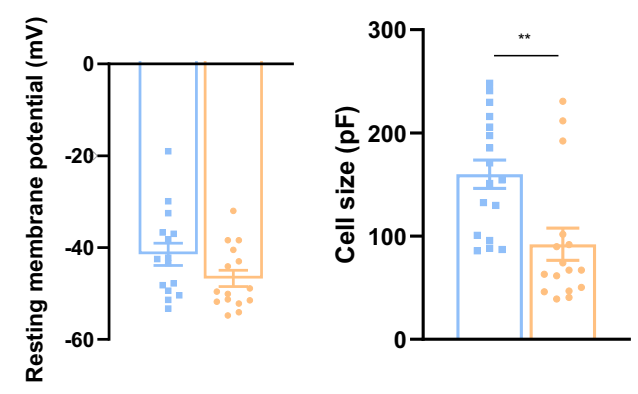

isoX9

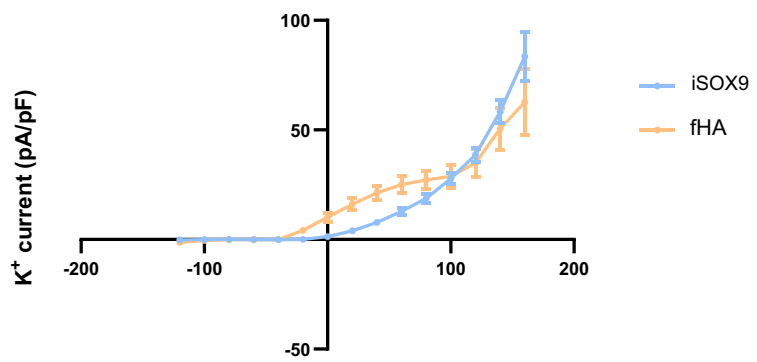

Voltage (mV)

d

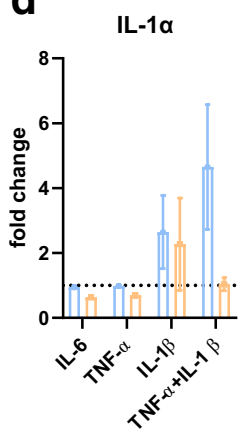

LIF

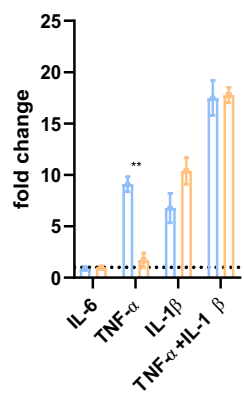

IFN- $\alpha$

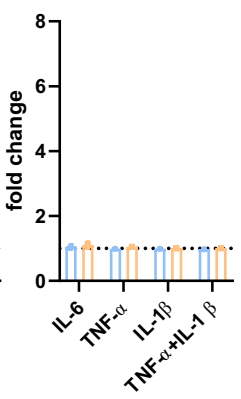

GM-CSF

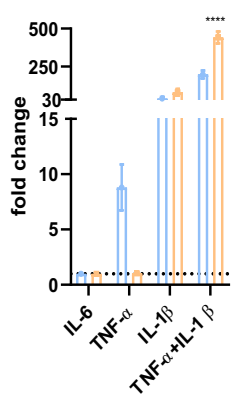

IFN- $\beta$

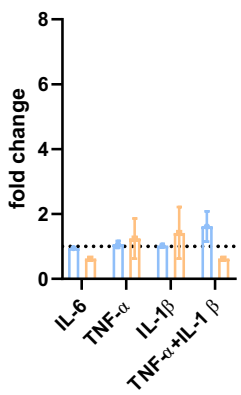

IL-6

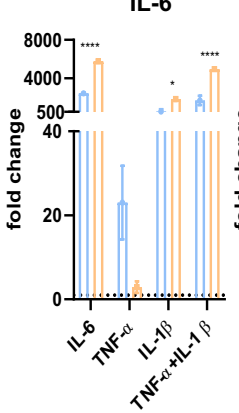

HGF

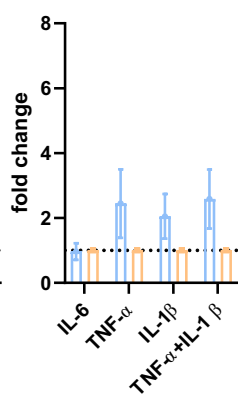

TNF- $\alpha$
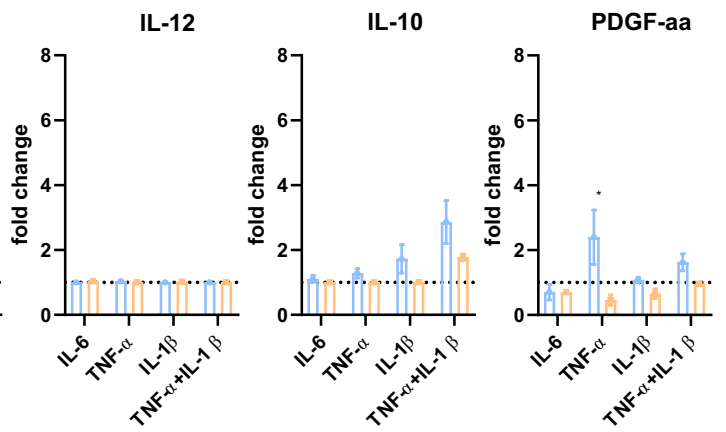

IL-1 $\beta$

IL-23
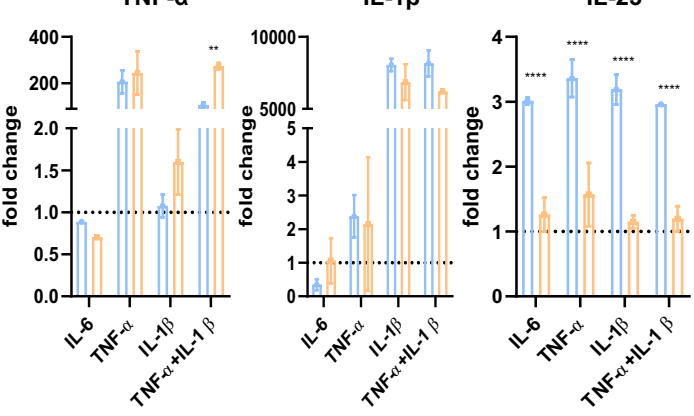

- isox9

$=\mathrm{fHA}$
Fig. 2 Functional characterization of iSOX9-astrocytes based on glutamate transport, patch clamp measurements and cytokine secretion. a Glutamate concentration was measured via mass spectrometry from the medium conditioned for 24,48 and 72 h by iSOX9astrocytes $(\mathrm{N}=8$ independent differentiations with two technical replicates for each differentiation) or fHA $(\mathrm{N}=4)$. In addition, medium with no cells was taken into account as negative control $(\mathrm{N}=4)(* * * \mathrm{p}<0.001$ versus fHA and iSOX9). b Resting membrane potential and cell capacitance (reflecting cell size) measurements of fHA ( $\mathrm{N}=15$ cells of 3 independent differentiations) and iSOX9-astrocytes $(\mathrm{N}=15$ cells of 3 independent differentiations $)(* * \mathrm{p}<0.01) . \mathrm{c} \mathrm{Na}^{+}$and $\mathrm{K}^{+}$current measurements via patch clamp at voltages between $-120 \mathrm{mV}$ and + $160 \mathrm{mV}$ of fHA $(\mathrm{N}=14)$ and iSOX9-astrocytes $(\mathrm{N}=17$ cells of 3 independent differentiations). d After exposing iSOX9-astrocytes $(\mathrm{N}=3$ independent differentiations with two technical replicates for each differentiation) and fHA $(\mathrm{N}=3)$ for 5 days to IL- 6 , TNF- $\alpha$, IL- $1 \beta$ or the combination of TNF- $\alpha$ and IL- $1 \beta$, the concentration of IL- $1 \alpha$, IFN- $\alpha$, IFN- $\beta$, HGF, IL-12, IL-10, LIF, GM-CSF, IL-6, TNF- $\alpha$, IL-1 $\beta$, PDGFaa and IL23 was measured in the medium using a bead-based immunoassay. Each time, the measured concentration was divided by the concentration of untreated cells $(* \mathrm{p}<0.05 ; * * \mathrm{p}<0.01 ; * * * \mathrm{*} p<0.0001)$. All data represented as mean \pm SEM 
a

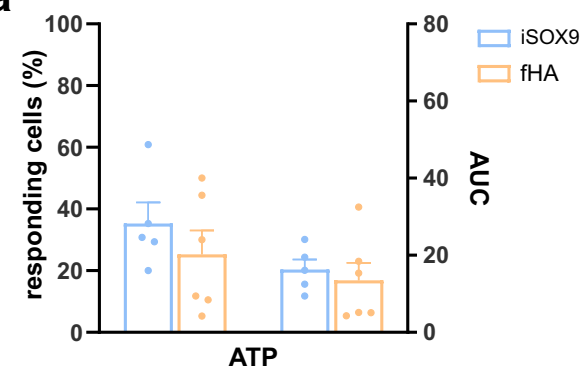

b

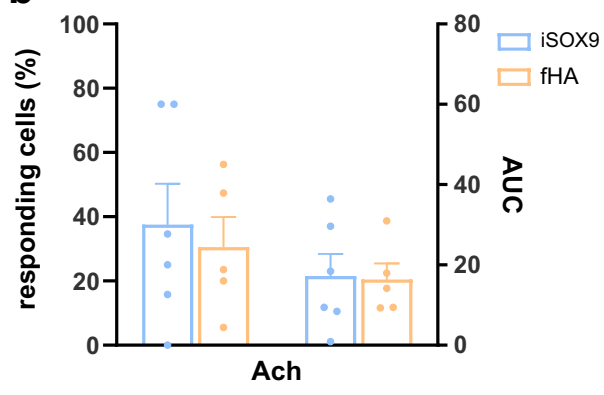

iSOX9

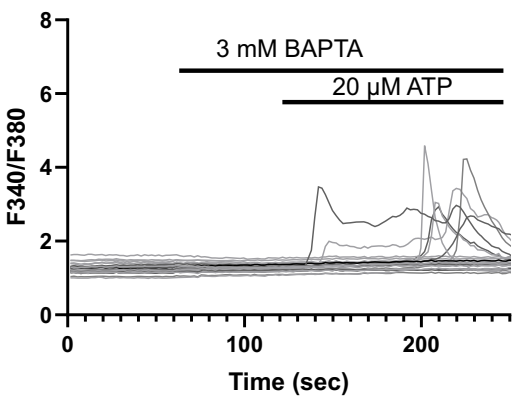

Time (sec)
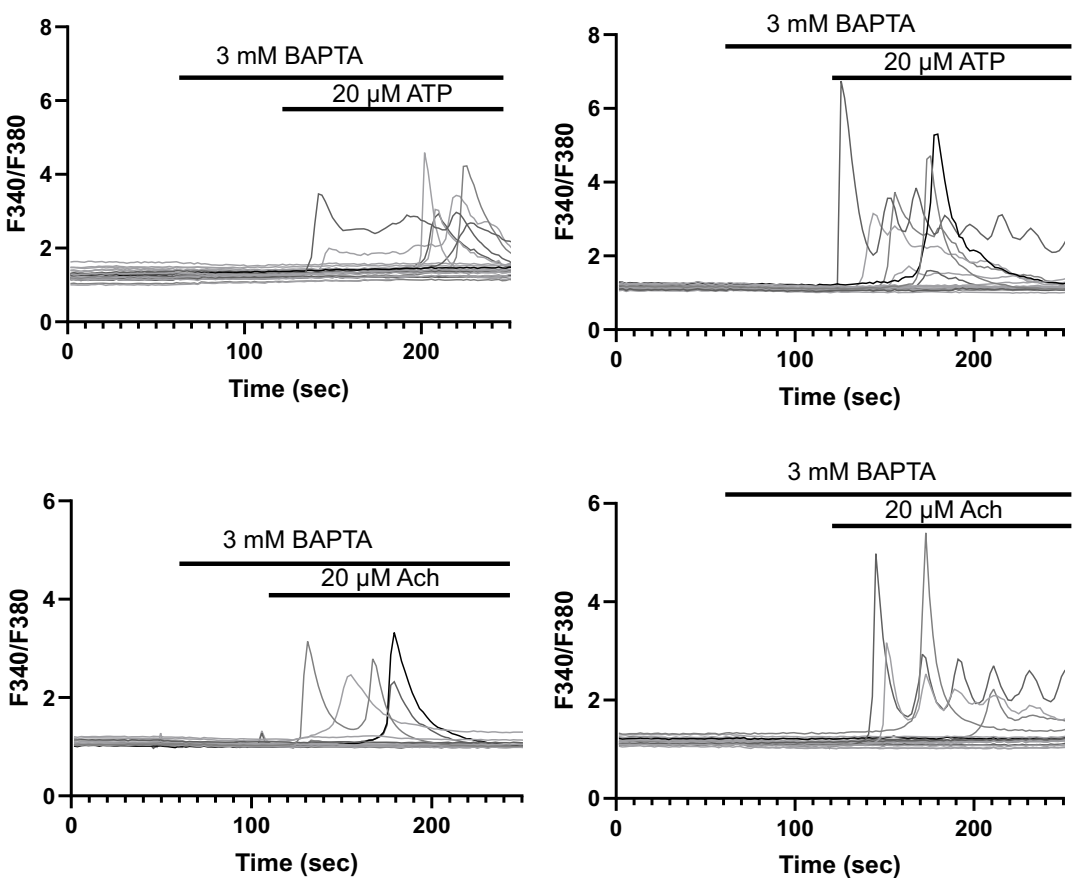

fHA

Time (sec)

C
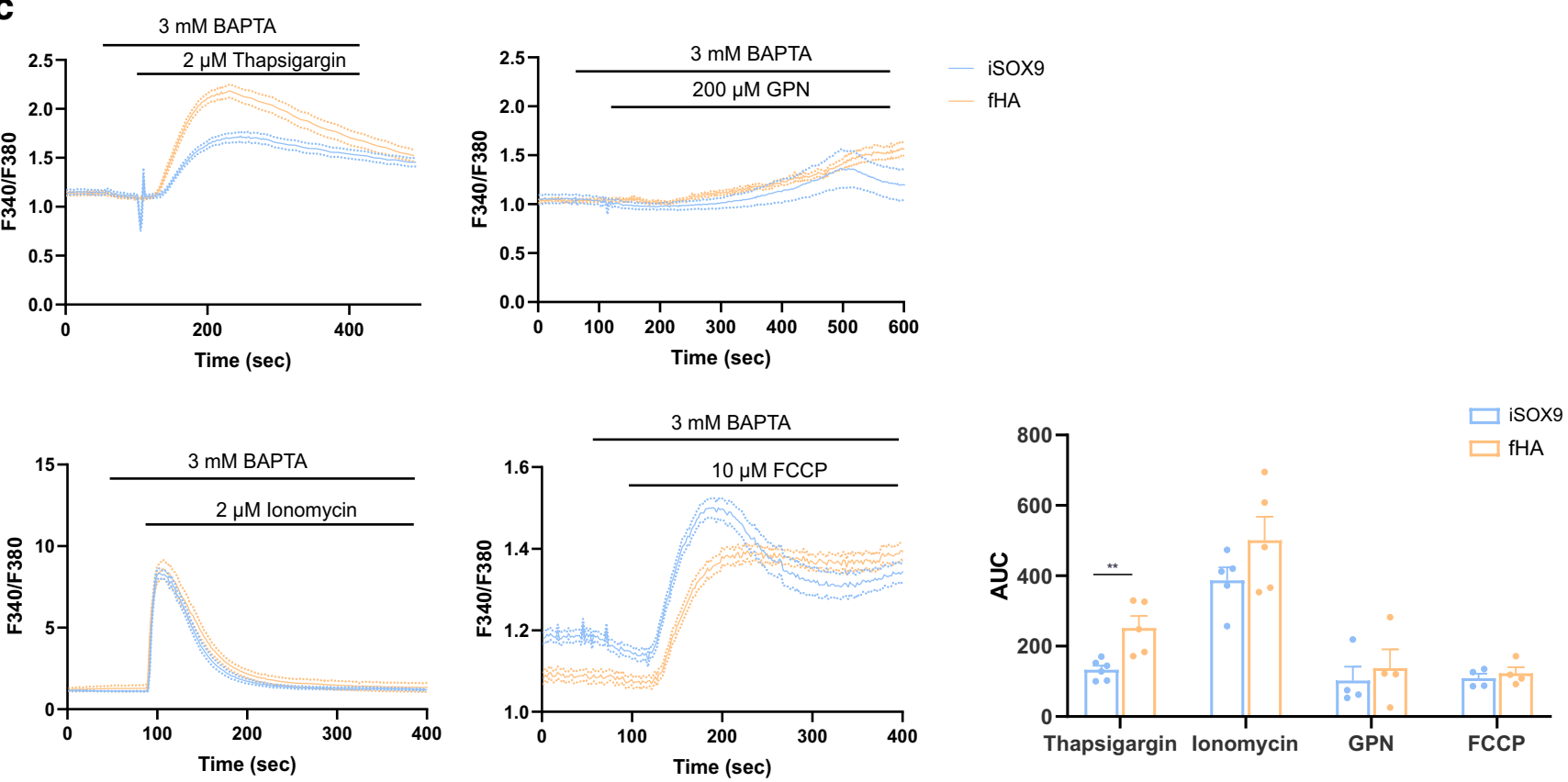

Fig. 3 iSOX9-astrocytes show comparable calcium responses as fHA. Calcium responses (plotted as F340/F380 ratio) measured in Fura-2loaded iSOX9-astrocytes and fHA in the presence of extracellular $\mathrm{Ca}^{2+}$ chelating agent BAPTA ( $3 \mathrm{mM})$, ensuring only $\mathrm{Ca}^{2+}$ release from internal $\mathrm{Ca}^{2+}$ stores is measured. a After stimulating fHA and iSOX9-astrocytes with ATP, they show a similar calcium response measured by Fura2 fluorescence emission ( $\mathrm{N}=6$ independent differentiations). b After stimulating fHA and iSOX9-astrocytes with Ach, they show a similar calcium

response measured by Fura2 fluorescence emission $(\mathrm{N}=6$ independent differentiations). c iSOX9-astrocytes and fHA show a similar calcium response after stimulation with GPN, FCCP and ionomycin measured by Fura2 fluorescence emission. A significantly higher calcium response is observed in fHA compared to iSOX9-astrocytes after thapsigargin stimulation $(* * \mathrm{p}<0.01)(\mathrm{N}=6$ independent differentiations). All data represented as mean \pm SEM

transcriptomes. When the PCA was repeated based on these 443 genes (Fig. 4c), we again found that culture-expanded fHA as well as iCell astrocytes clustered separately from iSOX9-astrocytes from both donors. This analysis also

demonstrated that all astrocytes generated in vitro by TF overexpression clustered together, and this in between freshly isolated fetal and postnatal astrocytes (Fig. 4c). Hierarchical clustering analysis of all samples (Fig. 4d) substantiated the notion 
a

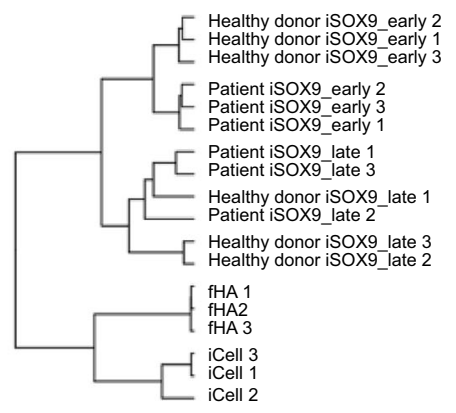

C

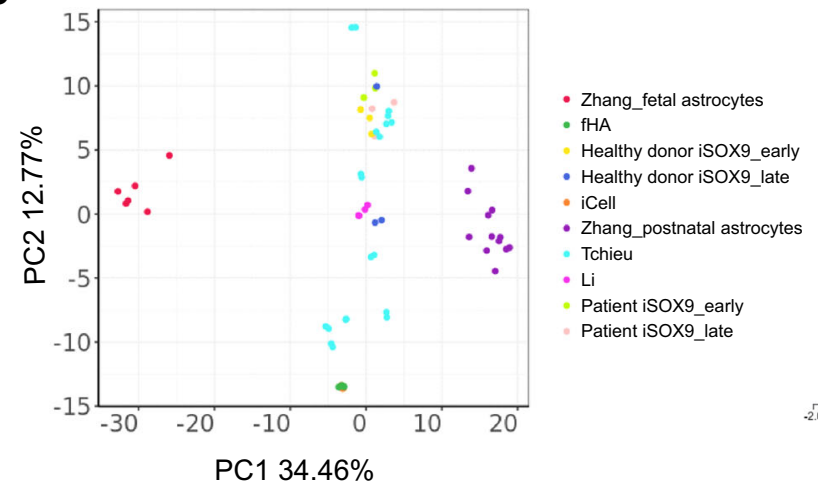

b

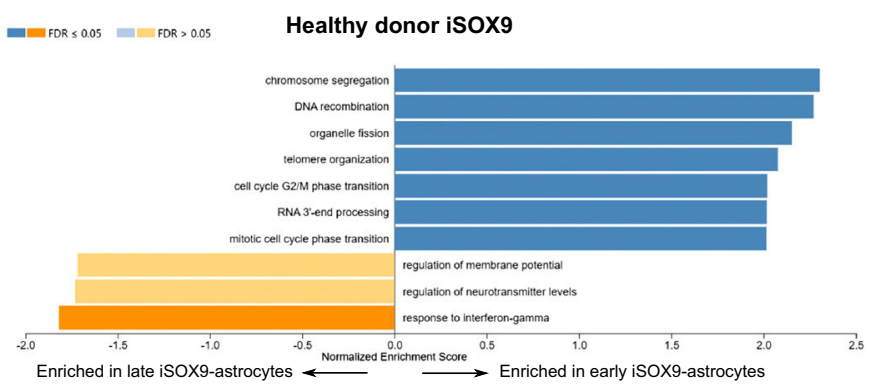

Patient iSOX9

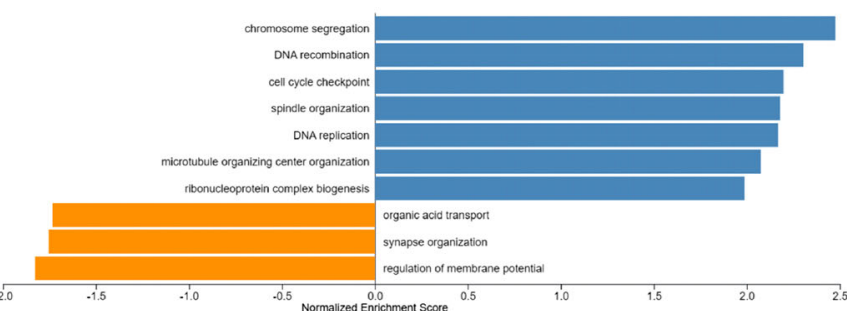

Enriched in late iSOX9-astrocytes $\stackrel{-0.5}{\longleftarrow} \stackrel{\text { Nomalized Enrichment score }}{\longrightarrow}$ Enriched in early iSOX9-astrocytes

d

Age/development related genes

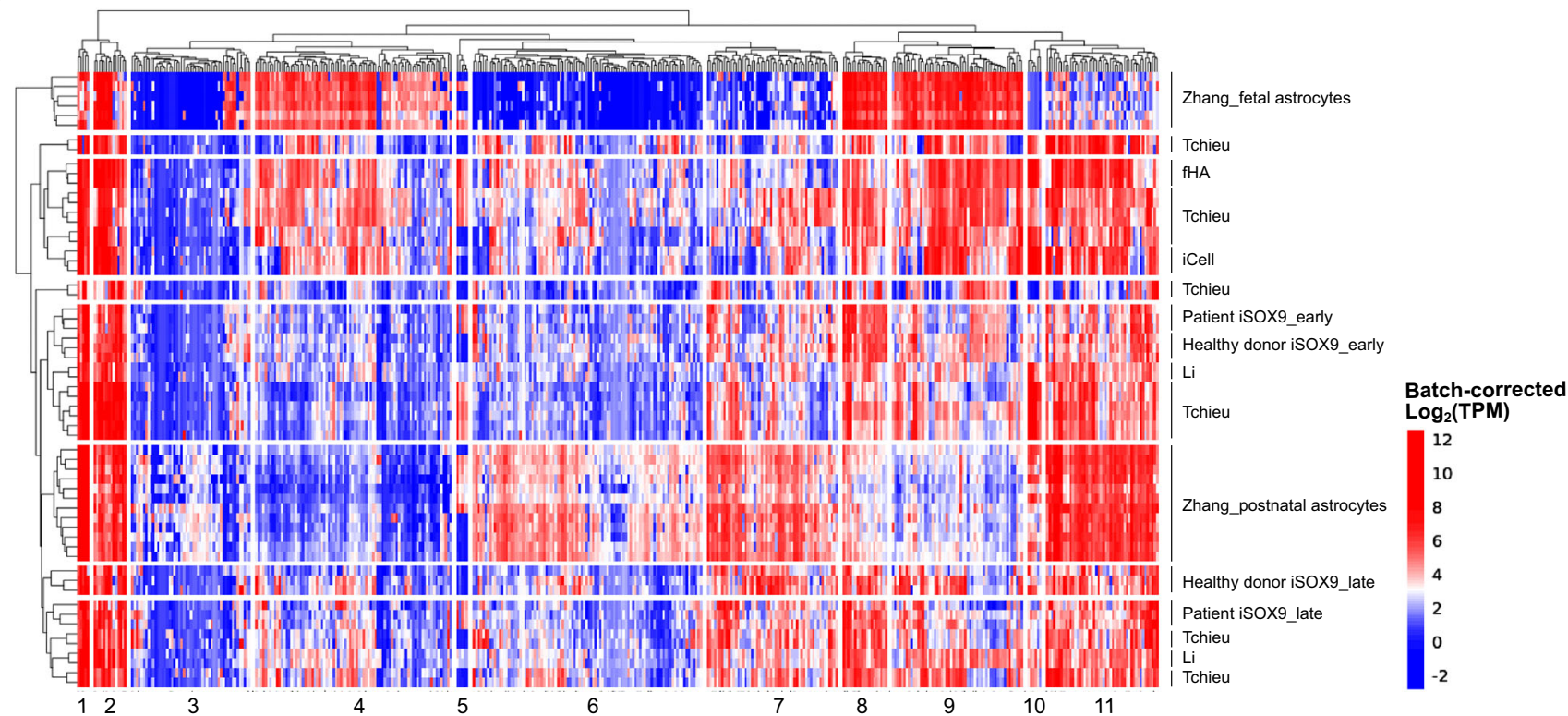

Fig. 4 RNASeq analysis of iSOX9-astrocytes reveals maturation after stopping doxycycline treatment and resemblance to isolated postnatal astrocytes. a Clustering of the iSOX9-astrocytes 5 (early) and 30 days (late) after stopping doxycycline treatment using a healthy donor-derived iPSC line and iPSCs derived from an ALS patient (each time $\mathrm{N}=3$ independent differentiations). Commercially available primary human fetal astrocytes (fHA) $(\mathrm{N}=3)$ and iPSC-derived astrocytes ("iCell") $(\mathrm{N}=3)$ were also included. b Ranked GSEA of the differentially expressed genes between early and late iSOX9-astrocytes. Biological processes with a positive normalized enrichment score (NES) (blue bars) are enriched in the early iSOX9-astrocytes, while the processes with a negative NES (orange bar) are enriched in the late iSOX9-astrocytes. This analysis was performed for both the healthy donor and patient-derived iPSC-derived cells. $\mathbf{c}$ PCA plot using only the genes that showed at least a 16-fold increased or decreased expression between the postnatal and fetal samples of Zhang et al., of the previous samples mentioned in panel (a) combined with RNASeq data from isolated postnatal and fetal human astrocytes from Zhang et al. and the iPSC-derived astrocytes from $\mathrm{Li}$ et al. and Tchieu et al. d Expression values of the genes that showed at least a 16-fold increased or decreased expression between the postnatal and fetal samples of Zhang et al. Those genes can be divided into 11 clusters and are considered important for development/age 
that iSOX9-astrocytes continue maturing when maintained longer in culture. Late iSOX9-astrocytes (both from healthy donor and patient) clustered closer together with postnatal than prenatal freshly isolated astrocytes. This was exemplified by lower levels of MKI67, TOP2A, TPX2 and NUSAP1, genes known to be highly expressed in astrocyte progenitor cells but downregulated upon maturation, both in late iSOX9astrocytes and postnatal astrocytes (Figure S6) [1]. Conversely, the mature astrocyte markers EAAT1, $A L D H 1 L 1$ and AQP4 were more highly expressed in late compared to early iSOX9-astrocytes and equally expressed compared to postnatal astrocytes (Figure S6). Hierarchical clustering analysis of the 443 genes identified 11 clusters (Fig. 4d). Clusters 4 and 9 represent genes involved in development and proliferation-related processes (e.g. "telencephalon development" and "nuclear division") and were expressed at higher levels in primary fetal astrocytes but at lower levels in postnatal isolated as well as iSOX9-astrocytes. Conversely, genes of cluster 6 and 11 comprising functional processes (e.g. "calcium ion transport" and "amino acid transport") were more highly expressed in postnatal compared with fetal isolated astrocytes, and remained lower expressed in all TFguided PSC-astrocyte populations (Fig. 4d and Figure S7a, $\mathrm{S} 7 \mathrm{~b})$.

\section{iSOX9-astrocytes Support iPSC-derived Neuron Maturation}

The most conclusive approach to assess the functional quality of human iPSC-derived astrocytes is by performing electrophysiological studies of iPSC-derived neurons cocultured with iPSC-derived astrocytes in a fully humanized assay set-up. To test the ability of iSOX9-astrocytes to support neuronal maturation and functioning, we cocultured them with iNGN2-neurons (as described in a recently submitted manuscript by Shih et al.). iNGN2derived NPCs were co-cultured together with DIV40 iSOX9-astrocytes on multi-electrode arrays (MEA) and readouts are shown after 37 days of co-culture (= DIV77). iSOX9-astrocyte mono-cultures were used as negative control, which did not display detectable electrical activity. We also added the comparison with primary rat neurons as the gold-standard reference used in electrophysiological studies. It has been previously shown that iNGN2-neurons on their own do not display electrical activity [49]. However, when co-cultured with iSOX9-astrocytes, we observed that the percentage of active electrodes and number of bursts significantly exceeded that of primary rat neurons $(\mathrm{p}<0.0001)$ (Fig. 5a, b). Importantly, the synchronicity index reflecting neural network formation was also significantly higher in iNGN2-neurons co-cultured with iSOX9-astrocytes than in primary rat neurons $(\mathrm{p}<0.0001)$ (Fig. 5c).

\section{Discussion}

In this study, we present a fast and efficient protocol to create human astrocytes from PSCs that support robust synchronous activity of PSC-derived iNGN2-neurons. This all-human PSC-derived astrocyte-neuron co-culture system represents an asset for high-throughput neural drug discovery studies, by which animal use may be reduced. However, complete removal of laboratory animals from research is not possible yet as animal models can capture the complex interactions in the neuronal system, which is not the case for these cocultures.

The protocol to generate large numbers of functional human astrocytes depends on the overexpression of $S O X 9$, as the sole TF, in DIV12 PSC-derived NPCs. Six days after doxycycline-induced SOX 9 overexpression, a nearly $100 \%$ pure EAAT1, ALDH1L1 and S100B positive cell population was obtained that could be further stably expanded for at least 30 days. iSOX9-astrocytes could be cryopreserved without loss of function after thawing as all mentioned functional read-outs were performed on both fresh and cryopreserved cells. Functionally, iSOX9-astrocytes displayed similar functional properties as cultured primary fetal human astrocytes (fHA). Transcriptionally, iSOX9-astrocytes showed similarities with postnatal brain astrocytes. As indicated above, iSOX9-astrocytes robustly supported the electrophysiological maturation of iNGN2-neurons.

Other groups already exploited TF-guided astrocyte differentiation to generate astrocytes from human PSCs [20-22]. Canals et al. transduced undifferentiated PSCs with lentiviral vectors (DIV1) encoding for TET-inducible $S O X 9$ and NFI-B, generating iAstrocytes after 21 days of doxycycline-induced overexpression of both TFs (DIV2-22). Although this protocol is shorter compared to ours (DIV33-48), the use of lentiviral vectors has the drawback that the copy number of either of the two viral vectors per cell likely varies. Moreover, silencing of viral vector integrants in longer term culture PSCs has been seen. Hence, because of variable vector integration and/or silencing, additional purification steps might be necessary at the end of differentiation. In addition, random integration of the vectors into the genome may lead to variable gene expression and viral vectors can cause insertional mutagenesis. Moreover, the protocol described by Canals et al. yielded only 1.8 iAstrocytes per starting PSC, while the method described here yields \pm 105 iSOX9-astrocytes per PSC. In a second study, Li et al. created PSCs wherein TET-inducible cDNAs for NFI-A and SOX9 were stably integrated into the AAVS1 locus of PSCs. In this study too, activation of the TFs via doxycycline was performed at the PSC stage creating astrocyte progeny 52 days later, which is longer than the iSOX9astrocyte protocol described here. In a third paper, Tchieu et al. also used a single TF overexpression system, by integrating a TET-inducible NFI-A in the AAVS1 locus of PSCs. 


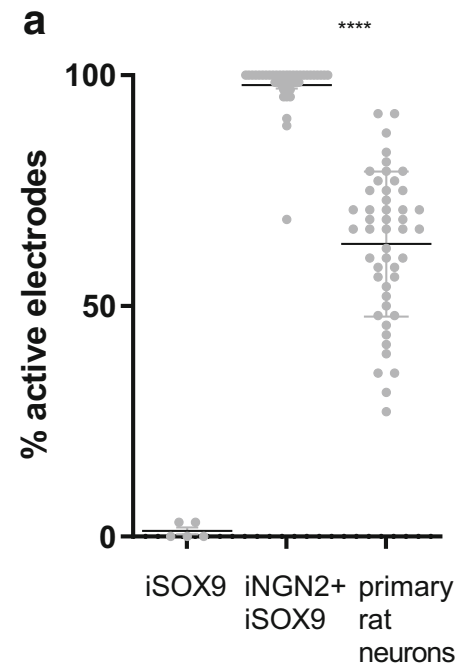

Fig. 5 iSOX9-astrocytes support iPSC-derived neuron maturation. DIV4 iNGN2-PSC-derived neurons were co-cultured 1:1 with DIV40 iSOX9astrocytes for 37 days and electrical activity was measured using MEA plates (16 electrodes per well of a 48 -well plate). As control, iSOX9-

After 20 days of neural induction (DIV20), NFI-A was induced by doxycycline addition for 10 days (DIV30), with a stable cell phenotype for another 30-60 days (DIV60-120) after doxycycline removal. In line with Tchieu et al., we observed that fewer than $50 \%$ of iSOX9-astrocytes expressed GFAP, which is significantly lower than what is seen for cultured fHA. However, GFAP gene expression in fHA is also considerably higher compared to the expression in freshly isolated fetal or adult astrocytes (Figure S6). As GFAP is expressed at low(er) levels in quiescent adult brain astrocytes but induced when astrocytes are activated [50,51], this may suggest that iSOX9-astrocytes have a less activated phenotype than fHAs. Of note, evaluation of the RNASeq studies perfomed by $\mathrm{Li}$ et al. and Tchieu et al. demonstrated similar NFI-A and SOX9 expression levels as observed in iSOX9astrocytes, even if we did not overexpress NFI-A as is done in either published studies (data not shown). This is in line with murine development studies demonstrating that SOX9 induces NFI-A expression and that the presence of both NFI$\mathrm{A}$ and $\mathrm{SOX} 9$ is required for astrocyte fating and maturation $[24,25]$.

To characterize the iSOX9-astrocytes, we compared their functional properties with those of fHA. Similar glutamate uptake was observed and also secretion of cytokines/growth factors in response to inflammatory signals was similar for iSOX9-astrocytes and fHA which is in line with previously published studies $[15,52,53]$. Electrophysiologically, iSOX9-astrocytes displayed a similar resting membrane potential and $\mathrm{Na}^{+}$and $\mathrm{K}^{+}$currents as fHA, although the inward $\mathrm{Na}^{+}$current was less pronounced in iSOX9-astrocytes than fHA. These patch clamp studies are consistent with the fact that the iSOX9-astrocytes are pure, and not contaminated by

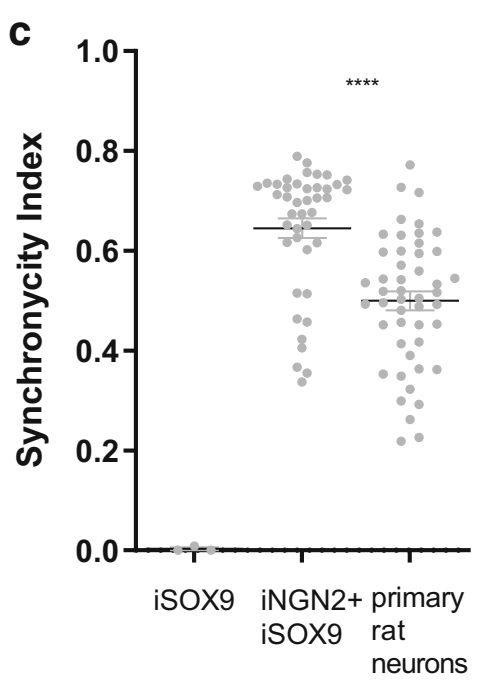

astrocytes alone and rat primary cortical neurons were also evaluated. Depicted are the \% of active electrodes (a), number of bursts (b) and synchronicity index $(\mathbf{c})$. Data is represented as mean \pm SEM of $N=3-4$ independent experiments

neurons, as the amplitude of $\mathrm{Na}^{+}$and $\mathrm{K}^{+}$currents measured in iPSC-derived neurons is at least 5 fold higher [45] than in the iSOX9-astrocytes. Spontaneous $\mathrm{Ca}^{2+}$ activity was not observed in the iSOX9-astrocytes, however intracellular $\mathrm{Ca}^{2+}$ release in vitro can be triggered in astrocytes by a number of inducers [54]. iSOX9-astrocytes as well as fHA responded in a similar manner to ATP and Ach as described before for primary rodent astrocytes [55, 56]. Compared to the differentiation protocol of Tchieu et al. in which $10 \%$ DIV 60 and $35 \%$ DIV 120 iAstrocytes responded to ATP exposure, we already observed $30 \%$ responding iSOX9-astrocytes by DIV48. Several reports described the response of mouse or rat astrocytes to thapsigargin [57, 58], $\operatorname{FCCP}[58,59]$, and ionomycin $[58,60]$. We found that treatment of iSOX9astrocytes with ionomycin, which measures the total amount of intracellular $\mathrm{Ca}^{2+}$ stored, was similar in iSOX9-astrocytes and fHA. However, the thapsigargininduced response, which releases $\mathrm{Ca}^{2+}$ from the ER, was lower in iSOX9-astrocytes compared with cultured fHA. As GPN and FCCP treatment showed similar $\mathrm{Ca}^{2+}$ responses in iSOX9-astroytes and fHA, the differences observed in thapsigargin-mediated release might suggest that the amount of $\mathrm{Ca}^{2+}$ stored in the Golgi may be higher in iSOX9-astrocytes. However, we anticipate that the significance of this difference for the use of these iSOX9astrocytes as model for drug development is low. ER $\mathrm{Ca}^{2+}$ store content is largely dependent on the amount of $\mathrm{Ca}^{2+}$ binding proteins present in the ER and/or overall ER size, which are expected to vary between individuals and as such may be more difficult to compare. More important is the observation that although the $\mathrm{ER} \mathrm{Ca}^{2+}$ store content was lower, the iSOX9-astrocytes display similar $\mathrm{Ca}^{2+}$ 
responses to physiological agonists that trigger $\mathrm{Ca}^{2+}$ release from the ER, such as ATP and acetylcholine.

Whole transcriptome analysis both of normal donor and ALS iSOX9-astrocytes demonstrated over time a decreased expression of astrocyte precursor cell genes [1] and increased expression of genes involved in neurotransmitter-related processes. Therefore, we suggest that the use of iSOX9-astrocytes at later timepoints (e.g. >DIV40) may be preferred in neuronal co-culture as the proliferation rate is low and such more mature astrocytes may better functionally support neurons. Transcriptionally, iSOX9-astrocytes displayed more similarities with freshly isolated postnatal astrocytes than prenatal astrocytes as described by Zhang et al. [39], which was more pronounced for DIV48 than DIV23 iSOX9-astrocytes. However, more in-depth studies are required to confirm this.

One of the most important functions of astrocytes is to provide metabolic and trophic support towards neurons [11], which in vitro translates to induction of functional maturation of neurons. Therefore, we assessed the neurophysiological properties of iPSC-derived neurons when co-cultured with iSOX9-astrocytes. Until now, variable effects of PSCderived astrocytes on neuronal maturation have been described via patch clamp $[20,22,61,62]$. For our study, neurons generated by the inducible overexpression of NGN2 from the AAVS1 locus in PSCs $[10,12]$ were used. Those iNGN2neurons on their own do not display electrical activity [49], however electrophysiological neuronal maturation is observed when co-cultured with PSC-derived astrocytes. Indeed, a recently submitted manuscript (Shih et al.) demonstrated that co-culture of DIV120 iPSC-derived astrocytes (generated via growth factors/small molecules) with iNGN2-neurons induced robust neuronal maturation. Here we demonstrated that the same iNGN2-neurons, when co-cultured with DIV40 iSOX9-astrocytes, acquire higher network activity measured by MEA analysis compared with rat neurons and iNGN2 cocultures with growth factor/cytokine-induced DIV120 astrocytes. The ability of performing co-cultures with iSOX9astrocytes already at DIV40 compared with the 120 days required in previous methods and their capacity to elicit optimal functional properties in human neurons, materializes a long standing objective of the stem cell field to create full humanbased neural models for drug discovery research in neurosciences.

The creation of a stable iSOX9-PSC line to overcome the drawbacks associated with the use of lentiviral vectors is perhaps less suitable in a disease modeling setting, as creation of the line is time consuming. In such case, SOX9 overexpression via lentiviral transduction of NPCs following dual SMAD inhibition might be more practical. However, we have observed that in some patient-derived iPSCs, NPCs are more vulnerable to the stress caused by viral transduction. Therefore, the transgenic AAVS1-located iSOX9-astrocyte differentiation system will mainly have an impact on fundamental astrocyte research as well as screening/drug discovery purposes in the neuroscience field. In addition, it should be noted that all functional read-outs were performed using only one iPSC line, namely SIGi001-A. However, in the transcriptome analysis ALS-patient derived iSOX9-astrocytes were also included which clustered closely together with the SIGi001-A-derived iSOX9-astroyctes. Moreover, we also introduced the inducible $S O X 9$ coding sequence in the AAVSI locus of H9 embryonic stem cells (ESCs) and based on RTqPCR and immunofluorescence, they could be similarly differentiated towards an astrocyte-like phenotype (Fig. S8).

In conclusion, we developed a robust, efficient and fast method to produce nearly $100 \%$ pure functional astrocytes starting from PSCs by overexpression of SOX9, as the sole TF, in PSC-derived NPCs. Already on DIV35-40, iSOX9astrocytes can be cryopreserved for downstream assays and phenocopy all functions of primary human astrocytes. Most importantly, iSOX9-astrocytes induce a higher degree of neuronal network formation in human PSC-derived neurons compared to gold-standard primary rat neuronal cultures. This high-yield protocol, requiring only \pm 40 days of differentiation, should now enable creating high-throughput all-human iPSC-derived astrocyte-neuron co-cultures suitable to support drug discovery.

Supplementary Information The online version contains supplementary material available at https://doi.org/10.1007/s12015-021-10179-x.

Acknowledgements We thank Prof. Ludo Van Den Bosch and Dr. Wenting Guo for providing the ALS patient-derived iPSC line (Laboratory of Neurobiology, Department of Neuroscience, VIB-KU Leuven). Furthermore, we would like to thank Dorien Broekaert (Laboratory of Cellular Metabolism and Metabolic Regulation, Department of Oncoloy, VIB-KU Leuven) for extracting and running the chromatography samples for the glutamate uptake experiment. Moreover, we thank the LIMONE core facility of VIB-KU Leuven Center for Brain and Disease Research to be able to use the Operetta CLS for imaging and thank Dr. Rodrigo Furtado Madeiro Da Costa for the help with the immunofluorescence quantification. Furthermore, we want to acknowledge the VIB nucleomics core (www.nucleomics.be) for processing, sequencing and providing the initial analysis of the RNA samples. We also express gratitude towards Christina Vochten and Vicky Raets for taking care of all the administration needed to conduct the research.

Author Contributions Katrien Neyrinck and Johanna Van Den Daele designed and performed all the experiments which were not carried out by collaborators. They analyzed and visualized the data of those experiments and wrote the manuscript. Tim Vervliet and Geert Bultynck helped design and perform the calcium imaging experiments and gave input about the interpretation of the data. Jonathan De Smedt integrated published RNASeq data into our data and helped with the interpretation of the transcriptomic data. Keimpe Wierda performed the astrocyte patch clamp experiments. Melissa Nijs helped differentiating the iSOX9-iPSCs towards astrocytes and conducting the RT-qPCR and immunofluorescence experiments. Tom Vanbokhoven helped during the lentiviral screen experiment and to clone the iSOX9 plasmid for RMCE. Astrid D'hondt 
helped with the generation of the iSOX9-iPSC lines. Sarah-Maria Fendt and Mélanie Planque helped designing and analyzing the glutamate uptake experiment. Pei-Yu Shih, Frederik Seibt, Juan Pita Almenar, Mohamed Kreir and Devesh Kumar performed and/or analyzed the electrophysiology co-culture studies. Vania Broccoli provided us with the idea and the needed plasmids to conduct this research. Alfredo CabreraSocorro performed the co-culture experiments on the MEA plates and, together with Andreas Ebnet, helped interpret all electrophysiological experiments. Catherine Verfaillie developed the research concept, guided the carried-out experiments and corrected the manuscript several times. All authors read and approved the final manuscript.

Funding This research is funded by the aspirant FWO grant for fundamental research of Katrien Neyrinck (application number 1166518 and $1166520 \mathrm{~N}$ ), the aspirant FWO grant for strategic basic research of Johanna Van Den Daele (SC/1S10717N) and a senior post doc FWO grant for Tim Vervliet (application number 12ZG121N). Furthermore, the research was funded by the Hercules grant AKUL/19/34 obtained by Geert Bultynck and Tim Vervliet and the following grants of which Catherine Verfaillie makes part: IWT.150031 iPSCAF, FWO.G0B5819N and from the EU/EFPIA/Innovative Medicines Initiative 2 Joint Undertaking (EBISC2 grant n ${ }^{\circ} 821362$ ).

\section{Declarations}

Conflict of Interest The authors declare that they have no known competing financial interests or personal relationships that could have appeared to influence the work reported in this paper.

Ethics Approval The ALS-patient derived iPSCs used in this study were generated from patient-derived skin biopsies approved by the ethical committee of the University Hospital Leuven (S50354).

\section{Consent to Participate Not applicable.}

\section{Consent for Publication Not applicable.}

Open Access This article is licensed under a Creative Commons Attribution 4.0 International License, which permits use, sharing, adaptation, distribution and reproduction in any medium or format, as long as you give appropriate credit to the original author(s) and the source, provide a link to the Creative Commons licence, and indicate if changes were made. The images or other third party material in this article are included in the article's Creative Commons licence, unless indicated otherwise in a credit line to the material. If material is not included in the article's Creative Commons licence and your intended use is not permitted by statutory regulation or exceeds the permitted use, you will need to obtain permission directly from the copyright holder. To view a copy of this licence, visit http://creativecommons.org/licenses/by/4.0/.

\section{References}

1. Vasile, F., Dossi, E., \& Rouach, N. (2017). Human astrocytes: structure and functions in the healthy brain. Brain Structure \& Function, 222(5), 2017-2029.

2. González-Reyes, R. E., Nava-Mesa, M. O., Vargas-Sánchez, K., Ariza-Salamanca, D., \& Mora-Muñoz, L. (2017). Involvement of astrocytes in Alzheimer's disease from a neuroinflammatory and oxidative stress perspective. Frontiers in Molecular Neuroscience, 10(December), 1-20.
3. Palmer, A. L., \& Ousman, S. S. (2018). Astrocytes and aging. Frontiers in Aging Neuroscience, 10(October), 1-14.

4. Wang, D. D., \& Bordey, A. (2008). The astrocyte odyssey. Progress in Neurobiology, 86(4), 342-367.

5. Jha, M. K., \& Morrison, B. M. (2018). Glia-neuron energy metabolism in health and diseases: New insights into the role of nervous system metabolic transporters. Experimental Neurology [Internet], 309(May):23-31. Available from: https://doi.org/10.1016/j. expneurol.2018.07.009.

6. Lee, S. W., Kim, W. J., Choi, Y. K., \& Kim, K. W. (2004). Oxygen regulates brain angiogenesis and tight junction formation in bloodbrain barrier. International Congress Series, 1262(C), 287-291.

7. Pfrieger, F. W. (2010). Role of glial cells in the formation and maintenance of synapses. Brain Research Reviews [Internet], 63(1-2):39-46. Available from: https://doi.org/10.1016/j. brainresrev.2009.11.002.

8. Min, R., \& van der Knaap, M. S. (2018). Genetic defects disrupting glial ion and water homeostasis in the brain. Brain Pathology, 28(3), 372-387.

9. Schousboe, A., Bak, L. K., \& Waagepetersen, H. S. (2013). Astrocytic control of biosynthesis and turnover of the neurotransmitters glutamate and GABA. Frontiers in Endocrinology (Lausanne), 4(AUG), 1-11.

10. Zhang, Y., Pak, C. H., Han, Y., Ahlenius, H., Zhang, Z., Chanda, S., et al. (2013). Rapid single-step induction of functional neurons from human pluripotent stem cells. Neuron, 78(5), 785-798.

11. Kuijlaars, J., Oyelami, T., Diels, A., Rohrbacher, J., Versweyveld, S., Meneghello, G., et al. (2016). Sustained synchronized neuronal network activity in a human astrocyte co-culture system. Science Reporter, 6(February 2016), 1-14.

12. Meijer, M., Rehbach, K., Brunner, J. W., Classen, J. A., Lammertse, H. C. A., van Linge, L. A., et al. (2019). A singlecell model for synaptic transmission and plasticity in human iPSC-derived neurons. Cell Reports, 27(7), 2199-2211.e6.

13. Taga, A., Dastgheyb, R., Habela, C., Joseph, J., Richard, J. P., Gross, S. K., et al. (2019). Role of human-induced pluripotent stem cell-derived spinal cord astrocytes in the functional maturation of motor neurons in a multielectrode array system. Stem Cells Translational Medicine, 8(12), 1272-1285.

14. Oberheim, N. A., Takano, T., Han, X., He, W., Lin, J. H. C., Wang, F., et al. (2009). Uniquely hominid features of adult human astrocytes. The Journal of Neuroscience, 29(10), 3276-3287.

15. Perriot, S., Mathias, A., Perriard, G., Canales, M., Jonkmans, N., Merienne, N., et al. (2018). Human induced pluripotent stem cellderived astrocytes are differentially activated by multiple sclerosisassociated cytokines. Stem Cell Reports, 11(5), 1199-1210.

16. Sloan, S. A., Darmanis, S., Huber, N., Khan, T. A., Birey, F., Caneda, C., et al. (2017). Human astrocyte maturation captured in $3 \mathrm{D}$ cerebral cortical spheroids derived from pluripotent stem cells. Neuron, 95(4), 779-790.e6.

17. di Domenico, A., Carola, G., Calatayud, C., Pons-Espinal, M., Muñoz, J. P., Richaud-Patin, Y., et al. (2019). Patient-specific iPSC-derived astrocytes contribute to non-cell-autonomous neurodegeneration in Parkinson's disease. Stem Cell Reports, 12(2), 213 229.

18. Oksanen, M., Petersen, A. J., Naumenko, N., Puttonen, K., Lehtonen, Š, Gubert Olivé, M., et al. (2017). PSEN1 mutant iPSC-derived model reveals severe astrocyte pathology in Alzheimer's disease. Stem Cell Reports, 9(6), 1885-1897.

19. Caiazzo, M., Giannelli, S., Valente, P., Lignani, G., Carissimo, A., Sessa, A., et al. (2015). Direct conversion of fibroblasts into functional astrocytes by defined transcription factors. Stem Cell Reports [Internet], 4(1):25-36. Available from: https://doi.org/10.1016/j. stemcr.2014.12.002.

20. Canals, I., Ginisty, A., Quist, E., Timmerman, R., Fritze, J., Miskinyte, G., et al. (2018). Rapid and efficient induction of 
functional astrocytes from human pluripotent stem cells. Nature Methods, 15(9), 693-696.

21. Li, X., Tao, Y., Bradley, R., Du, Z., Tao, Y., Kong, L., et al. (2018). Fast generation of functional subtype astrocytes from human pluripotent stem cells. Stem Cell Reports, 11(4), 998-1008.

22. Tchieu, J., Calder, E. L., Guttikonda, S. R., Gutzwiller, E. M., Aromolaran, K. A., Steinbeck, J. A., et al. (2019). NFIA is a gliogenic switch enabling rapid derivation of functional human astrocytes from pluripotent stem cells. Nature Biotechnology [Internet], 37(3):267-75. Available from: https://doi.org/10.1038/ s41587-019-0035-0.

23. Laug, D., Glasgow, S. M., \& Deneen, B. (2018). A glial blueprint for gliomagenesis. Nature Reviews Neuroscience [Internet], 19(7): 393-403. Available from: https://doi.org/10.1038/s41583-0180014-3.

24. Klum, S., Zaouter, C., Alekseenko, Z., Björklund, Å. K., Hagey, D. W., Ericson, J., et al. (2018). Sequentially acting SOX proteins orchestrate astrocyte- and oligodendrocyte-specific gene expression. EMBO Reports, 19(11), 1-14.

25. Stolt, C. C., Lommes, P., Sock, E., Chaboissier, M. C., Schedl, A., \& Wegner, M. (2003). The Sox9 transcription factor determines glial fate choice in the developing spinal cord. Genes \& Development, 17(13), 1677-1689.

26. Martini, S., Bernoth, K., Main, H., Ortega, G. D. C., Lendahl, U., Just, U., et al. (2013). A critical role for Sox9 in Notch-induced astrogliogenesis and stem cell maintenance. Stem Cells, 31(4), 741-751.

27. García-León, J. A., Kumar, M., Boon, R., Chau, D., One, J., Wolfs, E., et al. (2018). SOX10 single transcription factor-based fast and efficient generation of oligodendrocytes from human pluripotent stem cells. Stem Cell Reports [Internet], 10, 655-672. Available from: http://linkinghub.elsevier.com/retrieve/pii/ S2213671117305623.

28. García-León, J. A., García-Díaz, B., Eggermont, K., CáceresPalomo, L., Neyrinck, K., Madeiro da Costa, R., et al. (2020). Generation of oligodendrocytes and establishment of an allhuman myelinating platform from human pluripotent stem cells. Nature Protocols [Internet], 15(11):3716-44. Available from: https://doi.org/10.1038/s41596-020-0395-4.

29. Ordovás, L., Boon, R., Pistoni, M., Chen, Y., Wolfs, E., Guo, W., et al. (2015). Efficient recombinase-mediated cassette exchange in hPSCs to study the hepatocyte lineage reveals AAVS1 locusmediated transgene inhibition. Stem Cell Reports, 5(5), 918-931.

30. Martin, M. (2011). Cutadapt removes adapter sequences from highthroughput sequencing reads. EMBnet.journal, 17(1).

31. Morgan, M., Anders, S., Lawrence, M., Aboyoun, P., Pagès, H., Gentleman, R. ShortRead (2009). A bioconductor package for input, quality assessment and exploration of high-throughput sequence data. Bioinformatics, 25(19), 2607-2608.

32. Langmead, B., \& Salzberg, S. L. (2012). Fast gapped-read alignment with Bowtie 2. Nature Methods, 9(4), 357-359.

33. Dobin, A., Davis, C. A., Schlesinger, F., Drenkow, J., Zaleski, C., Jha, S., et al. (2013). STAR: Ultrafast universal RNA-seq aligner. Bioinformatics, 29(1), 15-21.

34. Li, H., Handsaker, B., Wysoker, A., Fennell, T., Ruan, J., Homer, N., et al. (2009). The sequence alignment/map format and SAMtools. Bioinformatics, 25(16), 2078-2079.

35. Liao, Y., Smyth, G. K., \& Shi, W. (2014). FeatureCounts: An efficient general purpose program for assigning sequence reads to genomic features. Bioinformatics, 30(7), 923-930.

36. Risso, D., Schwartz, K., Sherlock, G., \& Dudoit, S. (2011). GCcontent normalization for RNA-Seq data. BMC Bioinformatics, $12(1), 480+$

37. Robinson, M. D., \& Smyth, G. K. (2007). Moderated statistical tests for assessing differences in tag abundance. Bioinformatics, 23(21), 2881-2887.
38. Hochberg, Y. (2016). Controlling the false discovery rate: a practical and powerful approach to multiple testing author (s): Yoav Benjamini and Yosef Hochberg Source: Journal of the Royal Statistical Society Series B (Methodological), 57, No. 1 (1995), Publi. 57(1), 289-300.

39. Zhang, Y., Sloan, S. A., Clarke, L. E., Caneda, C., Plaza, C. A., Blumenthal, P. D., et al. (2016). Purification and characterization of progenitor and mature human astrocytes reveals transcriptional and functional differences with mouse. Neuron [Internet], 89(1):37-53. Available from: https://doi.org/10.1016/j.neuron.2015.11.013.

40. Elia, I., Broekaert, D., Christen, S., Boon, R., Radaelli, E., Orth, M. F., et al. (2017). Proline metabolism supports metastasis formation and could be inhibited to selectively target metastasizing cancer cells. Nature Communications, 8, 15267.

41. van Gorsel, M., Elia, I., Fendt, S. M. (2019). 13 C tracer analysis and metabolomics in 3D cultured cancer cells. Methods in Molecular Biology, 1862:53-66.

42. Lorendeau, D., Rinaldi, G., Boon, R., Spincemaille, P., Metzger, K., Jäger, C., et al. (2017). Dual loss of succinate dehydrogenase $(\mathrm{SDH})$ and complex I activity is necessary to recapitulate the metabolic phenotype of SDH mutant tumors. Metabolic Engineering [Internet], 43(November 2016):187-97. Available from: https:// doi.org/10.1016/j.ymben.2016.11.005.

43. Fernandez, C. A., Des Rosiers, C., Previs, S. F., David, F., \& Brunengraber, H. (1996). Correction of $13 \mathrm{C}$ mass isotopomer distributions for natural stable isotope abundance. Journal of Mass Spectrometry, 31(3), 255-262.

44. Mahmoud, S., Gharagozloo, M., Simard, C., \& Gris, D. (2019). Astrocytes maintain glutamate homeostasis in the CNS by controlling the balance between glutamate uptake and release. Cells, $8(2)$, 184.

45. García-León, J. A., Cabrera-Socorro, A., Eggermont, K., Swijsen, A., Terryn, J., Fazal, R., et al. (2018). Generation of a human induced pluripotent stem cell-based model for tauopathies combining three microtubule-associated protein TAU mutations which displays several phenotypes linked to neurodegeneration. Alzheimer's Dement, 14(10), 1261-1280.

46. Atakpa, P., Van Marrewijk, L. M., Apta-Smith, M., Chakraborty, S., \& Taylor, C. W. (2019). GPN does not release lysosomal Ca $2+$ but evokes $\mathrm{Ca} 2$ + release from the ER by increasing the cytosolic $\mathrm{pH}$ independently of cathepsin C. Journal of Cell Science, 132(3): jes 223883 .

47. Zhao, Z., Gordan, R., Wen, H., Fefelova, N., Zang, W. J., \& Xie, L. H. (2013). Modulation of intracellular calcium waves and triggered activities by mitochondrial $\mathrm{Ca}$ flux in mouse cardiomyocytes. PLoS One, 8(11), 1-17.

48. Guo, W., Naujock, M., Fumagalli, L., Vandoorne, T., Baatsen, P., Boon, R., et al. (2017). HDAC6 inhibition reverses axonal transport defects in motor neurons derived from FUS-ALS patients. Nature Communications [Internet], 8(1):1-14. Available from: https://doi. org/10.1038/s41467-017-00911-y.

49. Ho, S. M., Hartley, B. J., TCW, J., Beaumont, M., Stafford, K., Slesinger, P. A., et al. (2016). Rapid Ngn2-induction of excitatory neurons from hiPSC-derived neural progenitor cells. Methods [Internet], 101:113-24. Available from: https://doi.org/10.1016/j. ymeth.2015.11.019.

50. Placone, A. L., McGuiggan, P. M., Bergles, D. E., GuerreroCazares, H., Quiñones-Hinojosa, A., \& Searson, P. C. (2015). Human astrocytes develop physiological morphology and remain quiescent in a novel 3D matrix. Biomaterials, 42, 134-143.

51. Ridet, J. L., Malhotra, S. K., Privat, A., \& Gage, F. H. (1997). Reactive astrocytes: Cellular and molecular cues to biological function. Trends in Neurosciences, 20(12), 570-577.

52. Hyvärinen, T., Hagman, S., Ristola, M., Sukki, L., Veijula, K., Kreutzer, J., et al. (2019). Co-stimulation with IL-1 $\beta$ and TNF- $\alpha$ induces an inflammatory reactive astrocyte phenotype with 
neurosupportive characteristics in a human pluripotent stem cell model system. Scientific Reports, 9(1), 1-15.

53. Barbar, L., Jain, T., Zimmer, M., Kruglikov, I., Burstein, S., Rusielewicz, T., et al. (2020). CD49f is a novel marker to purify functional human iPSC-derived astrocytes. Neuron, 107(3), 436453.

54. Agulhon, C., Petravicz, J., McMullen, A. B., Sweger, E. J., Minton, S. K., Taves, S. R., et al. (2008). What is the role of astrocyte calcium in neurophysiology? Neuron, 59(6), 932-946.

55. Hamilton, N., Vayro, S., Kirchhoff, F., Verkhratsky, A., Robbins, J., Gorecki, D. C., et al. (2008). Mechanisms of ATP- and glutamate-mediated calcium signaling in white matter astrocytes. Glia, 56(7), 734-749.

56. Sharma, G., \& Vijayaraghavan, S. (2001). Nicotinic cholinergic signaling in hippocampal astrocytes involves calcium-induced calcium release from intracellular stores. Proceedings of the National Academy of Sciences of the United States of America, 98(7), 41484153.

57. Lange, J., Haslett, L. J., Lloyd-Evans, E., Pocock, J. M., Sands, M. S., Williams, B. P., et al. (2018). Compromised astrocyte function and survival negatively impact neurons in infantile neuronal ceroid lipofuscinosis. Acta Neuropathologica Communications, 6(1), 74.
58. Peuchen, S., Clark, J. B., \& Duchen, M. R. (1996). Mechanisms of intracellular calcium regulation in adult astrocytes. Neuroscience, 71(3), 871-883

59. Jackson, J. G., \& Robinson, M. B. (2015). Reciprocal regulation of mitochondrial dynamics and calcium signaling in astrocyte processes. The Journal of Neuroscience, 35(45), 15199-15213.

60. Müller, M. S., Obel, L. F., Waagepetersen, H. S., Schousboe, A., \& Bak, L. K. (2013). Complex actions of ionomycin in cultured cerebellar astrocytes affecting both calcium-induced calcium release and store-operated calcium entry. Neurochemical Research, 38(6), 1260-1265.

61. VanderWall, K. B., Vij, R., Ohlemacher, S. K., Sridhar, A., Fligor, C. M., Feder, E. M., et al. (2019). Astrocytes regulate the development and maturation of retinal ganglion cells derived from human pluripotent stem cells. Stem Cell Reports [Internet], 12(2):201-12. Available from: https://doi.org/10.1016/j.stemcr.2018.12.010.

62. Hedegaard, A., Monzón-Sandoval, J., Newey, S. E., Whiteley, E. S., Webber, C., \& Akerman, C. J. (2020). Pro-maturational Effects of Human iPSC-Derived Cortical Astrocytes upon iPSC-Derived Cortical Neurons. Stem Cell Reports, 15(1), 38-51.

Publisher's Note Springer Nature remains neutral with regard to jurisdictional claims in published maps and institutional affiliations. 